\title{
Molecular gas in distant brightest cluster galaxies
}

\author{
G. Castignani ${ }^{1}$, F. Combes ${ }^{2,3}$, P. Salomé ${ }^{2}$, and J. Freundlich ${ }^{4}$ \\ ${ }^{1}$ Laboratoire d'astrophysique, École Polytechnique Fédérale de Lausanne (EPFL), Observatoire de Sauverny, \\ 1290 Versoix, Switzerland \\ e-mail: gianluca.castignani@epfl.ch \\ 2 Sorbonne Université, Observatoire de Paris, Université PSL, CNRS, LERMA, 75014 Paris, France \\ 3 Collège de France, 11 place Marcelin Berthelot, 75231 Paris, France \\ 4 Centre for Astrophysics and Planetary Science, Racah Institute of Physics, The Hebrew University, Jerusalem 91904, Israel
}

Received 21 June 2019 / Accepted 23 December 2019

\begin{abstract}
The mechanisms governing the stellar mass assembly and star formation history of brightest cluster galaxies (BCGs) are still being debated. By means of new and archival molecular gas observations we investigate the role of dense megaparsec-scale environments in regulating the fueling of star formation in distant BCGs, through cosmic time. We observed in CO with the IRAM $30 \mathrm{~m}$ telescope two star-forming BCGs belonging to SpARCS clusters, namely, 3C $244.1(z=0.4)$ and SDSS J161112.65+550823.5 $(z=0.9)$, and compared their molecular gas and star formation properties with those of a compilation of $\sim 100$ distant cluster galaxies from the literature, including nine additional distant BCGs at $z \sim 0.4-3.5$. We set robust upper limits of $M_{\mathrm{H}_{2}}<1.0 \times 10^{10} M_{\odot}$ and $<2.8 \times 10^{10} M_{\odot}$ to their molecular gas content, respectively, and to the ratio of molecular gas to stellar mass $M\left(\mathrm{H}_{2}\right) / M_{\star} \lesssim 0.2$ and depletion time $\tau_{\text {dep }} \lesssim 40 \mathrm{Myr}$ of the two targeted BCGs. They are thus among the distant cluster galaxies with the lowest gas fractions and shortest depletion times. The majority $(64 \% \pm 15 \%$ and $73 \% \pm 18 \%)$ of the $11 \mathrm{BCGs}$ with observations in CO have lower $M\left(\mathrm{H}_{2}\right) / M_{\star}$ values and $\tau_{\text {dep }}$, respectively, than those estimated for main sequence galaxies. Statistical analysis also tentatively suggests that the values of $M\left(\mathrm{H}_{2}\right) / M_{\star}$ and $\tau_{\text {dep }}$ for the 11 BCGs deviates, with a significance of $\sim 2 \sigma$, from those of the comparison sample of cluster galaxies. A morphological analysis for a subsample of seven BCGs with archival HST observations reveals that $71 \% \pm 17 \%$ of the BCGs are compact or show star-forming components or substructures. Our results suggest a scenario where distant star-forming BCGs assemble a significant fraction $\sim 16 \%$ of their stellar mass on the relatively short timescale $\sim \tau_{\text {dep }}$, while environmental mechanisms might prevent the replenishment of gas feeding the star formation. We speculate that compact components also favor the rapid exhaustion of molecular gas and ultimately help to quench the BCGs. Distant star-forming BCGs are excellent targets for ALMA and for nextgeneration telescopes such as the James Webb Space Telescope.
\end{abstract}

Key words. galaxies: clusters: general - galaxies: star formation - galaxies: evolution - galaxies: active - molecular data

\section{Introduction}

Brightest cluster galaxies (BCGs) are excellent laboratories for studying the effect of dense galaxy cluster environments on galaxy evolution. In the local Universe they are commonly associated with passively evolving massive ellipticals of $\mathrm{cD}$ type, which often host radio galaxies (Zirbel 1996), and are located at the center of the cluster cores (Lauer et al. 2014), where tight correlations involving galaxy properties such as morphology (Dressler 1980), color (Kodama et al. 2001), stellar mass (Ostriker \& Tremaine 1975), star formation (Peng et al. 2010), and gas content (Gunn \& Gott 1972) are observed. Because of their exceptional masses and luminosities and because they are located in the crowded cores of clusters, BCGs are believed to evolve via phenomena such as dynamical friction (White 1976), galactic cannibalism (Hausman \& Ostriker 1978), interactions with the intracluster medium (Stott et al. 2012), and cooling flows (Salomé et al. 2006).

How the growth and star formation history of BCGs are regulated is still a matter of debate. Recent work suggests that they have doubled their stellar mass since $z \sim 1$ (Lidman et al. 2012), consistent with a global picture where BCGs evolve via dry accretion of satellite galaxies (Collins et al. 2009; Stott et al. 2011). More recent studies, however, have found potentially conflicting results to this somewhat simplistic hypothesis. Possible evidence for high star formation rates and large reservoirs of molecular gas in BCGs is found out to $z \sim 1$ and beyond (McDonald et al. 2016; Webb et al. 2015a,b; Bonaventura et al. 2017), thus favoring a scenario where star formation is fed by rapid gas deposition at high- $z$ and slow cooling flows at low- $z$ (Ocvirk et al. 2008; Dekel et al. 2009a,b).

Several studies have also found a high concentration of potentially in-falling, star-forming galaxies in the outskirts of nearby clusters (e.g., Bai et al. 2009; Chung et al. 2010), and a strong increase in the fraction of star-forming galaxies in cluster cores out to $z \sim 1$ and beyond (Smith et al. 2010; Tran et al. 2010; Tadaki et al. 2012; Webb et al. 2013; Brodwin et al. 2013; Zeimann et al. 2013; Santos et al. 2015; Alberts et al. 2016; Wang et al. 2016). Such findings seem to support the late assembly of cluster core members via the infall of gas-rich systems and via strong environmental quenching mechanisms (e.g., strangulation, ram pressure stripping, and galaxy harassment Larson et al. 1980; Moore et al. 1999).

On the other hand, other studies suggest the presence of a significant population of quiescent galaxies even in $z \gtrsim 1.5$ cluster cores (Tanaka et al. 2013; Koyama et al. 2014; Newman et al. 2014; Cooke et al. 2016; Strazzullo et al. 2016, 2019). In particular, Strazzullo et al. (2019) have recently studied the cluster 
galaxy population, including the BCGs, of a sample of five clusters at $z=1.4-1.7$ from the South-Pole Telescope Sunyaev Zel'dovich effect (SPT-SZ) survey (Bleem et al. 2015) and suggested that the star formation is suppressed in the cores of the massive clusters in their sample already earlier than $z \sim 1.5$.

Since molecular gas is an excellent tracer of ongoing or future star formation (Bigiel et al. 2008; Schruba et al. 2011; Leroy et al. 2013), observations of CO in both local and distant BCGs are a powerful tool that can be used to better understand the mass assembly and gas fueling of these sources. Some studies of BCGs in the local universe have, remarkably, found molecular gas reservoirs (Edge 2001; Salomé \& Combes 2003; Hamer et al. 2012; McNamara et al. 2014; Russell et al. 2014; Tremblay et al. 2016; Fogarty et al. 2019) and filaments of cold gas (Olivares et al. 2019; Russell et al. 2019).

In the literature, however, in the more distant Universe (i.e., $z>0.4$ ) there are only a handful of BCGs observed in CO (e.g., Webb et al. 2017; Emonts et al. 2013), mostly at $z>1$, where the average ratio of molecular gas to stellar mass in galaxies, at least in the field, is expected to increase significantly (by a factor of $\geq 4$ ) with respect to local galaxies (Carilli \& Walter 2013). To probe the star formation fueling of cluster core galaxies we recently performed a wide search of molecular gas in distant BCGs (Castignani et al. 2019) and cluster galaxies (Castignani et al. 2018) over a wide range of redshifts: $z \sim 0.4-2.6$. As part of this search, in this work we report $\mathrm{CO}$ observations of two other distant star-forming BCGs. The two BCGs span a broad range in cosmic time ( $3 \mathrm{Gyr}$ ), being located at $z=0.4$ and $z=0.9$, within which the overall molecular gas content of galaxies (Carilli \& Walter 2013) and their star formation activity (Madau \& Dickinson 2014) are expected to increase by a factor of $\sim 2$. With this work we thus complement our recent studies to reveal the fueling of gas feeding the star formation in the high- $z$ counterparts of present day starforming ( $>40 M_{\odot} \mathrm{yr}^{-1}$ ) BCGs, such as the famous Perseus A and Cygnus A galaxies (Fraser-McKelvie et al. 2014).

In this work we refer to proto-BCGs, BCGs, and BCG candidates with no distinction, keeping in mind that the secure identification of distant BCGs is difficult. This is ultimately due to the difficulty in confirming and characterizing high- $z$ cluster members and in particular the BCGs, which reside in crowded regions of the cluster cores and often have a complex (multicomponent) morphology.

The paper is structured as follows: in Sect. 2 we describe the two targets; in Sect. 3 we describe the observations and data reduction; in Sect. 4 we present the results; in Sect. 5 we summarize the results and draw our conclusions. In Appendix A we list the properties of distant cluster galaxies observed in CO. Throughout this work we adopt a flat $\Lambda \mathrm{CDM}$ cosmology with matter density $\Omega_{\mathrm{m}}=0.30$, dark energy density $\Omega_{\Lambda}=0.70$, and Hubble constant $h=H_{0} /\left(100 \mathrm{~km} \mathrm{~s}^{-1} \mathrm{Mpc}^{-1}\right)=0.70$ (but see Planck Collaboration VI 2018; Riess et al. 2019).

\section{Two brightest cluster galaxies}

We consider the Webb et al. (2015a) BCG catalog which comprises observed infrared (IR) properties of a large sample of 535 BCGs within the redshift range $0.2<z<1.8$. These BCGs belong to clusters drawn from the Spitzer Adaptation of the Red-Sequence Cluster Survey (SpARCS), which is an opticalnear-infrared galaxy-selected cluster survey, whose goal is to discover distant clusters out to $z \sim 2$ (Muzzin et al. 2009, 2012; Wilson et al. 2009; Demarco et al. 2010).
The authors provide both SFRs and stellar masses of the BCGs. The former were estimated from $24 \mu \mathrm{m}$ Spitzer-MIPS fluxes using the models by Chary \& Elbaz (2001) and the Kennicutt (1998) relation. The stellar masses were instead estimated from the observed $3.6 \mu \mathrm{m}$ flux, converted into the rest-frame $K$-band luminosity, taking the $\mathrm{K}$-correction into account.

As we want to target actively star-forming BCGs in order to investigate their molecular gas content and explore their evolution and interaction with their Mpc-scale environment, we consider spectroscopically confirmed sources from the Webb et al. (2015a) BCG catalog. This selection leaves us with 16 spectroscopically confirmed sources out of the 535 BCGs of the catalog. We also consider the subsample of five BCGs, at $0.4<z<1.1$, with the strongest star formation activity (i.e., star formation rate SFR $\left.>250 M_{\odot} \mathrm{yr}^{-1}\right)$. A sixth BCG at $z=1.7$ satisfies the selection requirements, but is not included because it has been already observed in $\mathrm{CO}(2 \rightarrow 1)$ by Webb et al. (2017), as discussed later in this work.

Of these five BCGs, we limited ourselves to two targets, namely 3C $244.1(z=0.4)$ and SDSS J161112.65+550823.5 $(z=$ 0.9 ), with $\mathrm{SFR}=281 M_{\odot} \mathrm{yr}^{-1}$ and $766 M_{\odot} \mathrm{yr}^{-1}$, respectively, for which we predicted to detect $\mathrm{CO}$ on the basis of standard relations by Tacconi et al. (2018), valid for main sequence (MS) field galaxies. The two BCGs belong to $M_{200} \gtrsim 1 \times 10^{14} M_{\odot}$ clusters and are located in the Lockman and ELAIS-N1 fields, respectively (Webb et al. 2015a). In particular, 3C 244.1 is a powerful Type II Fanaroff-Riley (FR) radio galaxy (Fanaroff \& Riley 1974) hosted by a cluster with an estimated richness of 15 galaxies within the cluster core (Hill \& Lilly 1991). Active galactic nucleus (AGN) contamination to the SFR estimates cannot be excluded, since the $24 \mu \mathrm{m}$ fluxes are associated with rest-frame emission at shorter wavelengths of 17 and $13 \mu \mathrm{m}$, respectively (see Sect. 4.5 for further discussion).

Some properties of the two sources are listed in Table 1, while in Fig. 1 we show their optical images taken from the data archives of the 12th release of SDSS ${ }^{1}$ and Pan-STARRS1 ${ }^{2}$. The images show that the two targeted BCGs are indeed bright, with i-band $\mathrm{AB}$ magnitudes of 18.0 (3C 244.1) and 21.5 (SDSS J161112.65+550823.5 ). A bulge-dominated morphology is also tentatively observed for 3 C 244.1, consistent with being the most massive galaxy in the cluster.

We used archival low-frequency $(<1 \mathrm{GHz})$ radio fluxes found in the NASA/IPAC Extragalactic Database (NED) to investigate some radio properties of the two BCGs. SDSS J161112.65 +550823.5 is a steep-spectrum radio source, with $\alpha=1.6$, while 3C 244.1 has a standard $\alpha=0.8$, typical of optically thin synchrotron emission in the jet. Here $\alpha$ denotes the low radio frequency spectral index, where the radio spectral flux density in units of Jy at the observer frame frequency $v$ is expressed as $S_{v} \propto v^{-\alpha}$. Using the spectral indexes and low-frequency radio fluxes, the rest-frame $408 \mathrm{MHz}$ luminosity densities $L_{408 \mathrm{MHz}}=$ $7.0 \times 10^{27} \mathrm{~W} \mathrm{~Hz}^{-1}$ and $1.5 \times 10^{25} \mathrm{~W} \mathrm{~Hz}^{-1}$ have been found for 3C 244.1 and SDSS J161112.65+550823.5, respectively. Therefore, 3C 244.1 has a low-frequency radio luminosity typical of powerful FR II sources, while SDSS J161112.65+550823.5 has a lower radio luminosity, more typical of the bulk of the radio galaxy population (Zirbel 1996). Both our targets are powerful radio sources, which strengthens the reliability of the BCG selection by Webb et al. (2015a). Distant radio galaxies have often been

\footnotetext{
1 https://skyserver.sdss.org/dr12/en/help/docs/ docshome. aspx

2 https://panstarrs.stsci.edu/
} 
Table 1. Properties of our targets, from Webb et al. (2015a).

\begin{tabular}{ccccccccc}
\hline \hline Galaxy ID & $\begin{array}{c}\text { RA } \\
\text { (hh:mm:ss.s })\end{array}$ & $\begin{array}{c}\text { Dec } \\
(\text { dd:mm:ss.s })\end{array}$ & $\begin{array}{c}z_{\text {spec }} \\
(2)\end{array}$ & $\begin{array}{c}L_{\mathrm{IR}} \\
\left(10^{11} L_{\odot}\right)\end{array}$ & $\begin{array}{c}M_{\star} \\
\left(10^{11} M_{\odot}\right)\end{array}$ & $\begin{array}{c}\text { SFR } \\
\left(M_{\odot} \mathrm{yr}^{-1}\right)\end{array}$ & $\begin{array}{c}\text { sSFR } \\
\left(\mathrm{Gyr}^{-1}\right)\end{array}$ & $\begin{array}{c}\text { sSFR } \\
\left(\mathrm{Gyr}^{-1}\right) \\
(8)\end{array}$ \\
\hline 3C 244.1 & $10: 33: 34.0$ & $+58: 14: 35.5$ & 0.430 & $16.1 \pm 1.2$ & 1.0 & $281 \pm 218$ & $2.8_{-2.5}^{+2.6}$ & 0.12 \\
SDSS J161112.65+550823.5 & $16: 11: 12.7$ & $+55: 08: 23.6$ & 0.907 & $43.7 \pm 15.8$ & 1.8 & $766 \pm 275$ & $4.3_{-2.9}^{+2.6}$ & 0.30 \\
\hline
\end{tabular}

Notes. The table lists: (1) Galaxy name; (2-3) J2000 equatorial coordinates; (4) spectroscopic redshift; (5) Total infrared luminosity inferred from $24 \mu \mathrm{m}$ observer frame Spitzer MIPS fluxes and using the Chary \& Elbaz (2001) model; (6) Stellar mass estimated using $3.6 \mu \mathrm{m}$ observer frame Spitzer IRAC fluxes, Bruzual \& Charlot (2003) stellar population modeling, and typical rest-frame $K$-band mass-to-light ratio of red galaxies (Bell et al. 2003); (7) SFR estimated from $L_{\mathrm{IR}}$ using the Kennicutt (1998) relation; (8) Specific SFR determined as sSFR = SFR/ $M_{\star}$; (9) sSFR for main sequence field galaxies with redshift and stellar mass of our targets estimated using the relation found by Speagle et al. (2014).

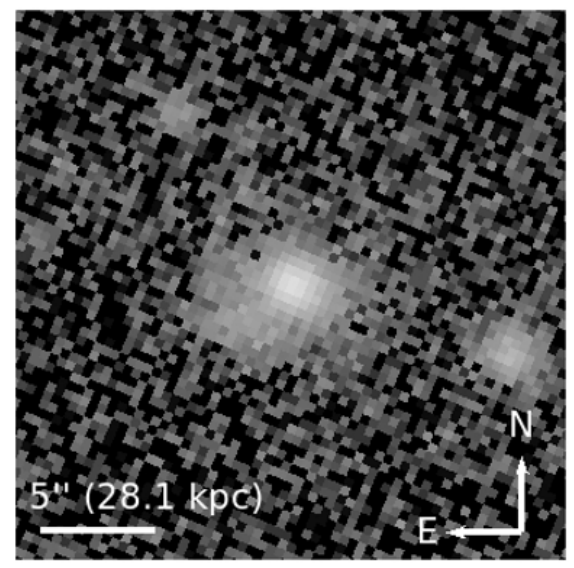

$3 \mathrm{C} 244.1$

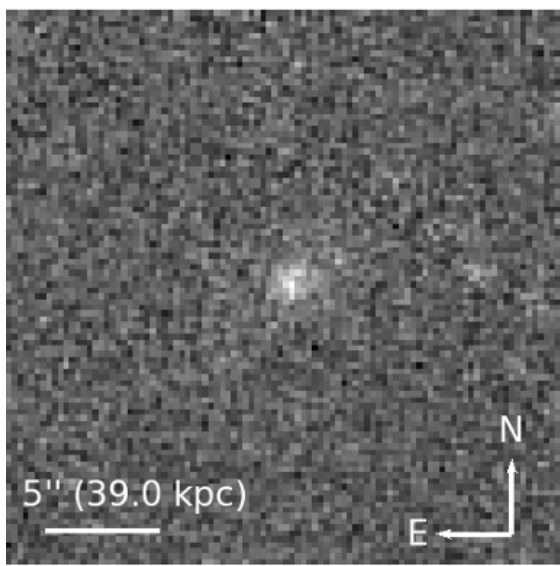

SDSS J161112.65+550823.5
Fig. 1. Optical images of the two target BCGs. Left: i-band SDSS DR12 image centered at the coordinates of 3C 244.1. Right: i-band PanSTARRS1 image centered at the coordinates of SDSS J161112.65+550823.5. Both images are $24^{\prime \prime} \times 24^{\prime \prime}$ in size. At the bottom left of each figure a $5^{\prime \prime}$ segment is shown, along with its corresponding value in physical units, at the redshift of the BCGs. found in association with BCG hosts (von der Linden et al. 2007; Yu et al. 2018; Castignani et al. 2019; Moravec et al. 2019).

\section{IRAM $30 \mathrm{~m}$ observations and data reduction}

We observed the two BCGs using the IRAM $30 \mathrm{~m}$ telescope at Pico Veleta in Spain. The observations of our targets were carried out between 3 and 6 July 2018, during the night, as part of the observational program 065-18 (P.I.: Castignani).

We used the Eight Mixer Receiver (EMIR) to observe $\mathrm{CO}(J \rightarrow J-1)$ emission lines from the target sources at frequencies between 81 and $242 \mathrm{GHz}$, corresponding to wavelengths between 3.0 and $1.2 \mathrm{~mm}$, where $J$ is a positive integer denoting the total angular momentum. For each source the specific $\mathrm{CO}(J \rightarrow J-1)$ transitions have been chosen to maximize the likelihood of the detection, in terms of the ratio of the predicted signal to the expected rms noise. In particular, we targeted simultaneously the $\mathrm{CO}(1 \rightarrow 0)$ and $\mathrm{CO}(2 \rightarrow 1)$ lines of $3 \mathrm{C} 244.1$ using the E090 and E150 receivers, respectively, while we targeted the $\mathrm{CO}(4 \rightarrow 3)$ line of SDSS J161112.65+550823.5 with the E230 receiver. We refer to Table 2 for further details.

The E090, E150, and E230 receivers can offer $4 \times 4 \mathrm{GHz}$ instantaneous bandwidth covered by the correlators. Of these four bands (UI, UO, LI, LO) we used only the lower side band LI for our CO search. The wobbler-switching mode was used for all the observations with a frequency of $0.5 \mathrm{~Hz}$ and a throw of $60 \mathrm{arcsec}$ to minimize the impact of atmosphere variability. The adopted wobbler throw is conservatively higher than the size of our target sources, which is in fact less than a few arcsec.
We originally planned to use the Wideband Line Multiple Autocorrelator (WILMA) to cover the LI-4 GHz band in each linear polarization. The WILMA back-end gives a resolution of $2 \mathrm{MHz}$; however, the WILMA was under maintenance during the observations. Therefore, we used only the fast Fourier transform spectrometers (FTSs) at $200 \mathrm{kHz}$ resolution to cover the $2 \times 4 \mathrm{GHz}$ lower sidebands (LI and LO) for each linear polarization. For SDSS J161112.65+550823.5 we also covered the $2 \times 4 \mathrm{GHz}$ upper sidebands (UI and UO) for each linear polarization.

The targets 3C 244.1 and SDSS J161112.65+550823.5 were observed for a total on-source observing time of $1.8 \mathrm{hr}$ and $3.9 \mathrm{hr}$, respectively. Observations were carried out in excellent weather conditions with an average precipitation water vapor (pwv) value of $\sim 3 \mathrm{~mm}$; average system temperatures $T_{\text {sys }}=106 \mathrm{~K}$ and $145 \mathrm{~K}$ for 3C 244.1 at $81 \mathrm{GHz}$ and $161 \mathrm{GHz}$, respectively; and $T_{\mathrm{sys}}=$ $204 \mathrm{~K}$ for SDSS J161112.65+550823.5 at $242 \mathrm{GHz}$.

Data reduction and analysis were performed using the CLASS software of the GILDAS package ${ }^{3}$. We also corrected the $\mathrm{CO}(1 \rightarrow 0)$ spectrum of $3 \mathrm{C} 244.1$ for a minor platforming level, corresponding to $0.85 \mathrm{mK}$ in $T_{\mathrm{a}}{ }^{*}$. The results are presented in Sect. 4.1.

M51 has been used as line calibrator, while Venus has been adopted to calibrate both pointing and focus. The following sources, when located at an elevation similar to those of our two targets, were used as pointing calibrators: IRC +10216 , PG 1418+546, 4C +39.25, 6C B104451.4+715930, 3C 454.3, and $3 \mathrm{C} 345$.

3 https://www.iram.fr/IRAMFR/GILDAS/ 
Table 2. Molecular gas properties.

\begin{tabular}{|c|c|c|c|c|c|c|c|c|c|}
\hline $\begin{array}{c}\text { Galaxy ID } \\
(1)\end{array}$ & $\begin{array}{l}z_{\text {spec }} \\
(2) \\
\end{array}$ & $\begin{array}{c}\mathrm{CO}(J \rightarrow J-1) \\
(3)\end{array}$ & $\begin{array}{c}v_{\mathrm{obs}} \\
(\mathrm{GHz}) \\
(4)\end{array}$ & $\begin{array}{c}S_{\mathrm{CO}(J \rightarrow J-1)} \\
\left(\mathrm{Jy} \mathrm{km} \mathrm{s}^{-1}\right) \\
(5)\end{array}$ & $\begin{array}{c}M\left(\mathrm{H}_{2}\right) \\
\left(10^{10} M_{\odot}\right) \\
(6)\end{array}$ & $\begin{array}{c}\tau_{\text {dep }} \\
\left(10^{8} \mathrm{yr}\right) \\
(7)\end{array}$ & $\begin{array}{c}\frac{M\left(\mathrm{H}_{2}\right)}{M_{\star}} \\
\left(10^{8} M_{\odot}\right) \\
(8)\end{array}$ & $\begin{array}{c}\tau_{\text {dep,MS }} \\
\left(10^{9} \mathrm{yr}\right) \\
(9)\end{array}$ & $\begin{array}{c}\left(\frac{M\left(\mathrm{H}_{2}\right)}{M_{\star}}\right)_{\mathrm{MS}} \\
(10)\end{array}$ \\
\hline 3C 244.1 & 0.430 & $\begin{array}{l}1 \rightarrow 0 \\
2 \rightarrow 1\end{array}$ & $\begin{array}{c}80.609 \\
161.215 \\
\end{array}$ & $\begin{array}{l}<0.95 \\
<0.77\end{array}$ & $\begin{array}{l}<4.0 \\
<1.0\end{array}$ & $\begin{array}{l}<1.4 \\
<0.36\end{array}$ & $\begin{array}{l}<0.40 \\
<0.10\end{array}$ & $1.09_{-0.14}^{+0.16}$ & $0.12_{-0.08}^{+0.19}$ \\
\hline SDSS J161112.65+550823.5 & 0.907 & $4 \rightarrow 3$ & 241.762 & $<0.93$ & $<2.8$ & $<0.37$ & $<0.16$ & $0.96_{-0.15}^{+0.18}$ & $0.25_{-0.13}^{+0.27}$ \\
\hline
\end{tabular}

Notes. The table lists: (1) Galaxy name; (2) Spectroscopic redshift as in Table 1; (3-4) $\mathrm{CO}(J \rightarrow J-1)$ transition and observer frame frequency; (5) $\mathrm{CO}(J \rightarrow J-1)$ velocity integrated flux; (6) Molecular gas mass; (7) Depletion timescale $\tau_{\text {dep }}=M\left(\mathrm{H}_{2}\right) / \mathrm{SFR}$; (8) Molecular gas-to-stellar mass ratio; (9-10) depletion timescale and molecular gas-to-stellar mass ratio predicted for MS field galaxies with redshift and stellar mass of our targets, following Tacconi et al. (2018). Upper limits are at $3 \sigma$.

\section{Results}

\subsection{Molecular gas properties}

We describe in this section the results obtained with the IRAM $30 \mathrm{~m}$ observations. Both targets were undetected by our observations. Based on the optical morphologies shown in Fig. 1 and assuming a CO-to-optical size ratio of $\sim 0.5$ (Young et al. 1995), the BCGs were also unresolved by our observations, with a beam of $\sim 10 \operatorname{arcsec}\left(\frac{242 \mathrm{GHz}}{v_{\mathrm{obs}}}\right)$ at observer frame frequency $v_{\mathrm{obs}}$ (Kramer et al. 2013).

We removed the baseline in each spectrum by using a polynomial fit of degree one. Then we estimated rms noise levels for the antenna temperature $\left(T_{\mathrm{A}}^{*}\right)$ equal to 0.18 and $0.13 \mathrm{mK}$ at $81 \mathrm{GHz}$ and $161 \mathrm{GHz}$, respectively, for 3C 244.1 , and $0.13 \mathrm{mK}$ at $242 \mathrm{GHz}$ for SDSS J161112.65+550823.5. These rms values were estimated within the entire LI- $4 \mathrm{GHz}$ bandwidth and at $300 \mathrm{~km} \mathrm{~s}^{-1}$ resolution. We then used the rms noise levels to set $3 \sigma$ upper limits.

In Table 2 we list the results of our analysis, where standard efficiency corrections have been applied to convert $T_{\mathrm{A}}^{*}$ into the main beam temperature $T_{\mathrm{mb}}$, and then $T_{\mathrm{mb}}$ into the corresponding $\mathrm{CO}$ line flux, where a $5 \mathrm{Jy} \mathrm{K}^{-1}$ conversion is used. We adopted the following efficiency corrections: $T_{\mathrm{mb}} / T_{\mathrm{A}}^{*}=1.17$, 1.27 , and 1.55 for 81,161 , and $242 \mathrm{GHz}$, respectively ${ }^{4}$.

To derive the $\mathrm{CO}(J \rightarrow J-1)$ luminosity $L_{\mathrm{CO}(J \rightarrow J-1)}^{\prime}$, in units of $\mathrm{K} \mathrm{km} \mathrm{s}^{-1} \mathrm{pc}^{2}$, from the velocity integrated $\mathrm{CO}(J \rightarrow J-1)$ flux $S_{\mathrm{CO}(J \rightarrow J-1)} \Delta v$, in units of Jy km s${ }^{-1}$, we used Eq. (3) of Solomon $\&$ Vanden Bout (2005):

$$
L_{\mathrm{CO}(J \rightarrow J-1)}^{\prime}=3.25 \times 10^{7} S_{\mathrm{CO}(J \rightarrow J-1)} \Delta v v_{\mathrm{obs}}^{-2} D_{L}^{2}(1+z)^{-3},
$$

where $v_{\mathrm{obs}}$ is the observer frequency in $\mathrm{GHz}$ of the $\mathrm{CO}(J \rightarrow$ $J-1)$ transition, $D_{\mathrm{L}}$ is the luminosity distance in $\mathrm{Mpc}$, and $z$ is the redshift of the galaxy.

By assuming a Galactic CO-to- $\mathrm{H}_{2}$ conversion factor $\alpha_{\mathrm{CO}}=$ $4.36 M_{\odot}\left(\mathrm{K} \mathrm{km} \mathrm{s}^{-1} \mathrm{pc}^{2}\right)^{-1}$, typical of MS galaxies (Solomon et al. 1997; Bolatto et al. 2013), for both targets we estimated $3 \sigma$ upper limits to the total molecular gas mass $M\left(\mathrm{H}_{2}\right)=\alpha_{\mathrm{CO}} L_{\mathrm{CO}(1 \rightarrow 0)}^{\prime}=$ $\alpha_{\mathrm{CO}} L_{\mathrm{CO}(J \rightarrow J-1)}^{\prime} / r_{\mathrm{J} 1}$. Here $r_{\mathrm{J} 1}=L_{\mathrm{CO}(J \rightarrow J-1)}^{\prime} / L_{\mathrm{CO}(1 \rightarrow 0)}^{\prime}$ is the excitation ratio. We assumed the following fiducial excitation ratios, namely $r_{21}=0.8$ (typical of distant star-forming galaxies, Bothwell et al. 2013; Daddi et al. 2015; Freundlich et al. 2019) and $r_{41}=0.4$ (Papadopoulos et al. 2000). As summarized in Appendix A, the adopted excitation ratios and CO-to- $\mathrm{H}_{2}$ conversion factors are consistent with those typically used in the literature for distant star-forming galaxies in clusters. Being located

\footnotetext{
4 https://wWw.iram.es/IRAMES/mainWiki/

Iram30mEfficiencies
}

in the cluster cores, the target BCGs live in the densest regions of the clusters where the gas can be easily excited. As further discussed in Sect. 4.5, the use of a lower CO-to- $\mathrm{H}_{2}$ conversion factor, more appropriate for star-forming galaxies above the MS, does not substantially impact our final results. We also note that $\mathrm{CO}(J \rightarrow J-1)$ lines with $J>2$ may not be optimal $\mathrm{H}_{2}$ tracers since they probe denser gas than lower $J=1,2 \mathrm{CO}$ transitions. However, at increasing redshift lower $J$ transitions become fainter and are redshifted towards lower frequencies, while higher transitions $(J>2)$ enter the millimeter domain and have indeed been used as $\mathrm{H}_{2}$ tracers in several studies (see Appendix A).

We did not attempt to set an upper limit to the continuum emission of the target galaxies by using the available total FTS $8 \mathrm{GHz}$ (LI, LO) and $16 \mathrm{GHz}$ (LI, LO, UI, UO) bandwidths associated with 3C 244.1 and SDSS J161112.65+550823.5, respectively, for each polarization. The faintness of our targets, the significant intrinsic atmospheric instability at millimeter wavelengths, and the platforming occurring with the FTS backend (see also Sect. 3) prevented us from determining robustly the continuum level or from estimating its upper limit.

We used the SFRs and our molecular mass estimates to set $3 \sigma$ upper limits to the depletion timescale associated with the consumption of the molecular gas and equal to $\tau_{\text {dep }}=$ $M\left(\mathrm{H}_{2}\right) / \mathrm{SFR}$. Similarly, we also set $3 \sigma$ upper limits to the molecular gas-to-stellar mass ratio $M\left(\mathrm{H}_{2}\right) / M_{\star}$. For comparison, we computed the depletion time $\tau_{\text {dep,MS }}$ and the molecular gasto-stellar mass ratio $\left(\frac{M\left(\mathrm{H}_{2}\right)}{M_{\star}}\right)_{\mathrm{MS}}$ for MS field galaxies with redshift and stellar mass equal to those of our target galaxies, as found using empirical prescriptions by Tacconi et al. (2018).

\subsection{Comparison sample of distant (proto-)cluster galaxies}

We compared the results found for our sources in terms of stellar mass, SFR, molecular gas content, and depletion time with those found in the literature for distant cluster galaxies with both stellar mass estimates and CO observations. In particular, we considered the reference sample of $0.2 \lesssim z \lesssim 5.0$ cluster galaxies observed in $\mathrm{CO}$ and described in our recent work (Castignani et al. 2019, and references therein).

We summarize the comparison sample in the following. It includes (proto-)cluster galaxies at $z \sim 0.2$ (Cybulski et al. 2016); $z \sim 0.4-0.5$ (Geach et al. 2011; Jablonka et al. 2013); $z \sim 1.1-1.2$ (Wagg et al. 2012; Castignani et al. 2018); $z \sim 1.5-1.7$ (Aravena et al. 2012; Rudnick et al. 2017; Webb et al. 2017; Noble et al. 2017, 2019; Hayashi et al. 2018; Kneissl et al. 2019); $z \sim 2.0-2.5$ (Emonts et al. 2013, 2016; Ivison et al. 2013; Tadaki et al. 2014; Dannerbauer et al. 2017; Lee et al. 2017; Coogan et al. 2018; Wang et al. 2018); $z \sim 0.4-2.6$ (Castignani et al. 2019); $z \sim 3.47$ 
(Candels-5001, Ginolfi et al. 2017); $z=4.05$ (Tan et al. 2014); $z=5.2-5.3$ (Walter et al. 2012; Riechers et al. 2010).

We updated this sample by replacing the results found by Dannerbauer et al. (2017) and Tadaki et al. (2014) for the $z \simeq 2.2$ Spider Web and $z \simeq 2.5$ USS 1558-003 protocluster galaxies, respectively. In this work we adopt the updated results by Tadaki et al. (2019), who reported a total of $10 \mathrm{CO}(3 \rightarrow 2)$ detections for these two protoclusters. We also include for the present analysis the submillimeter galaxy $\mathrm{J} 221735.15+001537.3$ detected in $\mathrm{CO}(3 \rightarrow 2)$ by Bothwell et al. (2013) and belonging to the protocluster SSA22 at $z=3.10$ for which both stellar mass and SFR estimates are available (Umehata et al. 2015). We also considered eight additional galaxies, with stellar mass estimates, which have been recently detected in CO by Gómez-Guijarro et al. (2019). Half of the sources have been observed in $\mathrm{CO}(3 \rightarrow 2)$ and belong to the HELAISS02 protocluster $(z=2.171)$, while the other four have been observed in $\mathrm{CO}(1 \rightarrow 0)$ and belong to the HXMM20 protocluster $(z=2.602)$.

This selection yields 118 sources over 33 (proto-)clusters. By also including 3C 244.1 and SDSS J161112.65+550823.5 the final sample comprises 120 sources, all with $M_{\star}>10^{9} M_{\odot}$, and over 35 (proto-)clusters at $0.2 \lesssim z \lesssim 5.0$.

Including $\mathrm{SFR} \gtrsim 3 \times \mathrm{SFR}_{\mathrm{MS}}$ sources might result in biasedhigh molecular gas masses (see, e.g., Noble et al. 2017; Castignani et al. 2018, 2019, for discussion). However, since our target sources have estimated SFR $\gtrsim 10 \times \mathrm{SFR}_{\mathrm{MS}}$ we prefer not to discard such high-SFR galaxies from the comparison.

\subsection{Distant BCGs observed in CO}

Among the $\sim 100$ distant sources with CO observations and stellar mass estimates considered in the previous section there are only a few BCG candidates. As discussed in Castignani et al. (2019) they are i) the $z=1.7 \mathrm{BCG}$ observed in $\mathrm{CO}(2 \rightarrow 1)$ by Webb et al. (2017), which belongs to the Webb et al. (2015a) sample of distant BCGs, similarly to our two targets; ii) the $z=1.99$ BCG candidate of the cluster ClJ1449+0856, detected in $\mathrm{CO}(4 \rightarrow 3)$ and $\mathrm{CO}(3 \rightarrow 2)$ by Coogan et al. (2018); iii) MRC 1138-262 at $z=2.2$ (i.e., the Spider Web galaxy), detected in $\mathrm{CO}(1 \rightarrow 0)$ by Emonts et al. $(2013,2016)$; and iv) Candels5001 at $z=3.472$, detected in $\mathrm{CO}(4 \rightarrow 3)$ by Ginolfi et al. (2017).

In addition to these four sources, in Castignani et al. (2019) we recently reported $\mathrm{CO}$ observations for five additional distant BCG candidates hosting radio galaxies. The sources were selected within the DES SN deep fields and COSMOS survey as part of a large search for distant $z>0.3$ star-forming radio galaxies. Our analysis yielded upper limits to the total $\mathrm{H}_{2}$ gas mass for the five radio sources, namely DES radio galaxies 399, 708, COSMOS-FR I 16, 31, and 70 at redshifts $z=0.39,0.61$, $0.97,0.91$, and 2.63 , respectively.

By including 3C 244.1 and SDSS J161112.65+550823.5 from this work, our final BCG subsample consists of 11 distant BCG candidates observed in $\mathrm{CO}$, spanning a broad redshift range $z \sim 0.4-3.5$. Consistently with the value adopted for 3C 244.1 and SDSS J161112.65+550823.5 (see Sect. 4.1) to allow a homogeneous comparison throughout this work, we assume a Galactic CO-to- $\mathrm{H}_{2}$ conversion factor $\alpha_{\mathrm{CO}}=$ $4.36 M_{\odot}\left(\mathrm{K} \mathrm{km} \mathrm{s}^{-1} \mathrm{pc}^{2}\right)^{-1}$.

In Appendix A we summarize redshifts, stellar masses, and molecular gas properties for the compilation of 120 (proto-) cluster galaxies observed in $\mathrm{CO}$, at different transitions and with different telescopes. While we adopt a constant $\alpha_{\mathrm{CO}}$ to convert velocity integrated $\mathrm{CO}(1 \rightarrow 0)$ luminosities into $M\left(\mathrm{H}_{2}\right)$ masses, for each source we choose the excitation ratio reported in the corresponding reference study. We refer to Table A.1 for details, while in Table A.2 we focus only on the 11 BCG candidates. The tables show that the sample of distant cluster galaxies observed in $\mathrm{CO}$, remarkably, has increased fourfold since 2017. For comparison, we refer to the recent compilation by Dannerbauer et al. (2017, Table A.1) of $z>0.4$ cluster galaxies.

In the cases where uncertainties for the SFR and $M_{\star}$ are not found in the reference studies we assumed a fiducial $50 \%$ error for the SFR and $\sim 0.3$ dex uncertainty for $M_{\star}$. Stellar mass estimates commonly rely on stellar population synthesis models and have statistical uncertainties $\sim(0.10-0.14)$ dex (e.g., Roediger \& Courteau 2015). An additional $~ 0.25$ dex uncertainty may be added because of the unknown initial mass function (e.g., Wright et al. 2017, and references therein), resulting in a typical $\sim 0.3$ dex uncertainty on $M_{\star}$, which is also adopted for our two target BCGs.

\subsection{Molecular gas, star formation, and depletion time}

In Fig. 2 we show the ratio of molecular gas to stellar mass $M\left(\mathrm{H}_{2}\right) / M_{\star}$ as a function of both redshift and specific SFR for the $\sim 100$ distant sources with CO observations and stellar mass estimates. The 11 distant BCGs are highlighted. In Fig. 3 we show the SFR, $M\left(\mathrm{H}_{2}\right) / M_{\star}$, and the depletion time $\left(\tau_{\text {dep }}\right)$, all normalized to their MS values, as a function of the stellar mass.

These plots show that the BCGs indeed populate the highmass end of the galaxy population with $\mathrm{CO}$ observations, having stellar masses in the range $\log \left(M_{\star} / M_{\odot}\right)=10.3-12.0$. Given the large uncertainties and scatter in the data points, the BCGs also have SFRs and $M\left(\mathrm{H}_{2}\right) / M_{\star}$, both of which are consistent with the values found using empirical relations for the MS (Tacconi et al. 2018; Speagle et al. 2014) and with those of the other distant cluster galaxies observed in $\mathrm{CO}$.

However, 7 out of the 11 BCGs (i.e., the majority, $64 \% \pm$ $15 \%)^{5}$ have $M\left(\mathrm{H}_{2}\right) / M_{\star}$ values that are lower than those estimated for MS galaxies of similar stellar mass and redshift by using the Tacconi et al. (2018) relation. This also applies to our targets 3C 244.1 and SDSS J161112.65+550823.5. Furthermore COSMOS-FRI 16 and 31 at $z \simeq 1.0$ have upper limits to the $M\left(\mathrm{H}_{2}\right) / M_{\star}$ ratio that are higher than the MS values. Therefore, it may also be that the fraction of BCGs with low values of $M\left(\mathrm{H}_{2}\right) / M_{\star}$ is even higher than estimated. Similarly, 6 out of the 11 BCGs (i.e., the majority, $55 \% \pm 15 \%$ ) have SFRs that are higher than those estimated for MS galaxies of similar stellar mass and redshift by adopting the Speagle et al. (2014) relation.

Interestingly, combining these results for the $M\left(\mathrm{H}_{2}\right) / M_{\star}$ values and the SFR we obtain that the 11 considered BCGs have, overall, low depletion times $\tau_{\text {dep }}=M\left(\mathrm{H}_{2}\right) / \mathrm{SFR}$, with estimated values or upper limits in the range (0.04-7) Gyr. As can be seen in Fig. 3 (right), eight BCGs (i.e., $73 \% \pm 18 \%$ ) have indeed $\tau_{\text {dep }}$ values that are lower than those, $\tau_{\text {dep,MS }}$, estimated for the MS using the Tacconi et al. (2018) relation. Limiting ourselves to the remaining three BCGs, the Spider Web galaxy has large uncertainties associated with the depletion time, while COSMOSFRI 16 and 31 only have upper limits to the $M\left(\mathrm{H}_{2}\right) / M_{\star}$ ratio. These results suggest that the large majority or possibly all 11 BCGs have $\tau_{\text {dep }} \lesssim \tau_{\text {dep,Ms }}$.

\footnotetext{
5 Here and throughout the text the uncertainties on the percentage $(1 \sigma)$ are estimated using the binomial distribution. We refer to Castignani et al. (2014) for a similar methodology.
} 

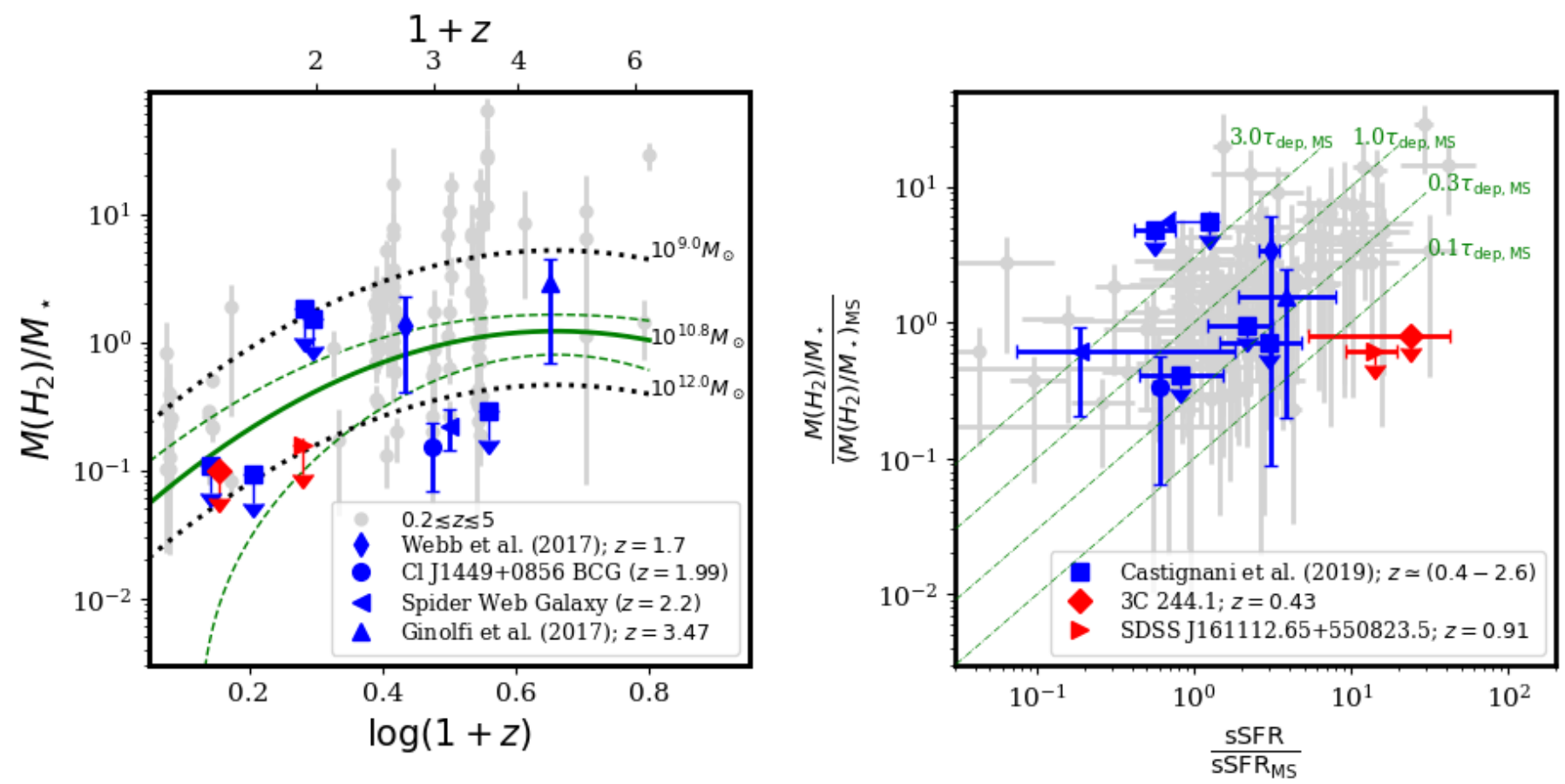

Fig. 2. Molecular gas properties of distant BCGs and cluster galaxies observed in CO. Left: evolution of the molecular gas-to-stellar mass ratio as a function of the redshift for cluster galaxies at $0.2 \lesssim z \lesssim 5$ observed in CO. The solid green curve is the scaling relation found by Tacconi et al. (2018) for field galaxies in the MS and with $\log \left(M_{\star} / M_{\odot}\right)=10.8$. The green dashed lines show the statistical $1 \sigma$ uncertainties in the model. The dotted black lines show the same scaling relation as the solid green line, but for different stellar masses, $\log \left(M / M_{\star}\right)=9$ and 12 , which correspond to the stellar mass range spanned by the data points. Right: molecular gas-to-stellar mass ratio vs. the specific SFR for the cluster galaxies in our sample, both normalized to the corresponding MS values using the relations for the ratio and the SFR by Tacconi et al. (2018) and Speagle et al. (2014), respectively. The dot-dashed green lines show different depletion times, in units of the depletion time at the MS. The legend for the data points is shown in the bottom right corner of each panel.
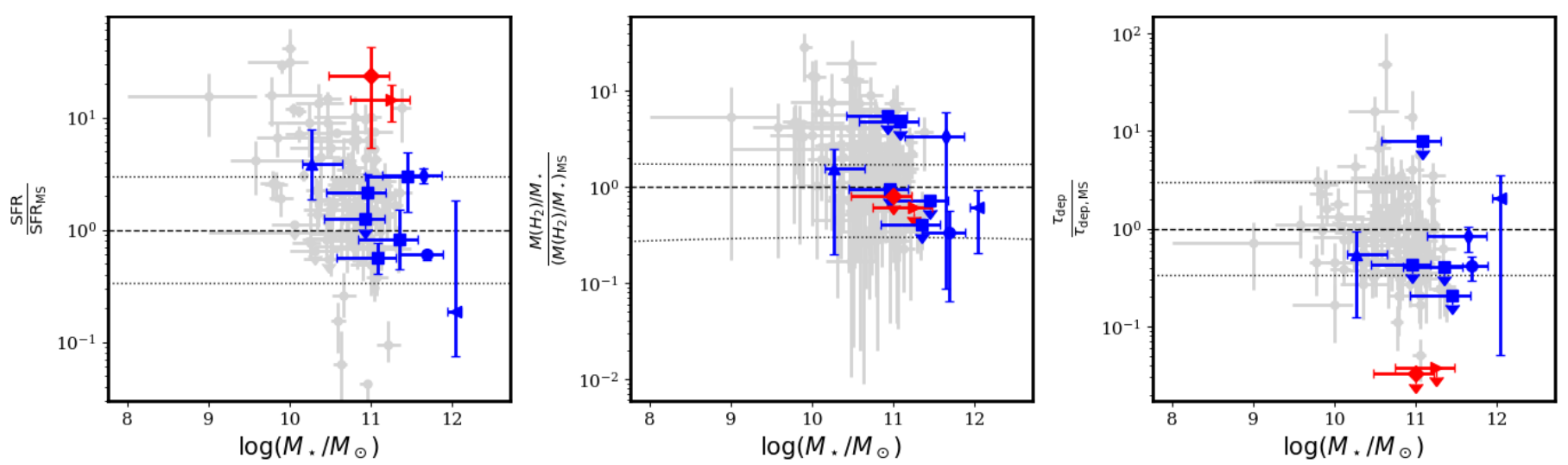

Fig. 3. Star formation rate (left), molecular gas-to-stellar mass ratio (center), and depletion time (right), as a function of the stellar mass for distant BCGs and cluster galaxies observed in CO. The $y$-axis values are all normalized to the corresponding MS values using the relations by Speagle et al. (2014) and Tacconi et al. (2018). The horizontal dashed lines correspond to $y$-axis values equal to unity, while the dotted lines denote the fiducial uncertainties associated with the MS. The uncertainty is chosen equal to \pm 0.48 dex for both left and right panels, since the MS is commonly identified by $1 / 3<\mathrm{SFR} / \mathrm{SFR}_{\mathrm{MS}}<3$. For the central panel, the plotted uncertainties are estimated at redshift $z=1$. The color-coding for the data points is the same as in Fig. 2.

We also compared the properties of the 11 BCGs with those of the comparison sample by means of the Kolmogorov-Smirnov test. The null hypothesis for the $\log \left(M_{\star} / M_{\odot}\right)$ distributions of the two populations is rejected with a significance of $3.2 \sigma$, consistent with the fact that BCGs are indeed the most massive galaxies in the clusters. When considering the $M_{\mathrm{H}_{2}} / M_{\star}$, $\left(M_{\mathrm{H}_{2}} / M_{\star}\right) /\left(M_{\mathrm{H}_{2}} / M_{\star}\right)_{\mathrm{MS}}$, and $\tau_{\text {dep }} / \tau_{\text {dep,MS }}$ distributions for the two populations, significances of $2.4 \sigma, 1.7 \sigma$, and $2.0 \sigma$ are found, respectively. These results suggest that there is a tentative evidence at $\sim 2 \sigma$ that the population of distant star-forming BCGs differ with respect to that of distant star-forming cluster galaxies in terms of their molecular gas content and depletion time, while no statistically significant difference in terms of their $\mathrm{SFR} / \mathrm{SFR}_{\mathrm{MS}}$ has been found.

Interestingly, given the high SFR (on average) associated with the 11 BCGs, they are also expected to assemble a significant fraction $f=\tau_{\text {dep }} \mathrm{SFR} / M_{\star}$ of their stellar mass within the timescale $\sim \tau_{\text {dep }}$. By considering upper limits to the depletion 
time as true measurements, we find a median value of $f \simeq 16 \%$. Similarly, McDonald et al. (2014) find that a significant fraction $f \simeq 3 \%$ of the stellar mass is provided for the Phoenix $\mathrm{A}$ $\mathrm{BCG}$ at $z=0.597$ within its starburst phase $\left(\mathrm{SFR} \sim 800 \mathrm{M}_{\odot} \mathrm{yr}^{-1}\right.$, McDonald et al. 2013) in a short $\tau_{\text {dep }} \sim 30$ Myr. Therefore, nonnegligible $f$-values and relatively short $\tau_{\text {dep }}$ seem to be common for distant star-forming BCGs, quite independently of their redshifts, $z \simeq 0.4-3.5$, corresponding to a 7.4 Gyr interval in cosmic time.

Our results, based on observations in $\mathrm{CO}$ of distant $z \sim 0.4-3.5$ star-forming BCGs, are consistent with a broader picture where environmental mechanisms (e.g., strangulation, ram pressure stripping, and galaxy harassment) tend to favor the exhaustion of the BCG gas reservoirs on a relatively short timescale $\sim \tau_{\text {dep }}$. Furthermore, while our study considers a subsample of rare star-forming BCGs and might not be of general validity for the entire BCG population in the distant Universe, it also suggests a scenario where star-forming BCGs assemble a significant fraction of their stellar mass not only during early stages $(z \gtrsim 2)$, but also at later epochs $(z \lesssim 1)$. Our results thus support recent studies that proposed that BCGs grow by a factor of $\sim 2$ in stellar mass since $z \sim 1$ (Lidman et al. 2012; Zhang et al. 2016), but are also not in contradiction with theoretical predictions, according to which most of the BCG stellar mass is assembled at higher redshifts $(z \sim 3-5)$ in smaller sources which will later be swallowed by the BCGs (De Lucia \& Blaizot 2007).

\subsection{AGN contamination and SFR}

Further considerations are needed concerning the SFR estimates of the 11 BCGs. They have $1 / 5 \lesssim \mathrm{SFR} / \mathrm{SFR}_{\mathrm{MS}} \lesssim 4$, with the exception represented by the two IRAM $30 \mathrm{~m}$ targets of this work, for which SFR/SFR ${ }_{M S} \sim 10-20$. Such high SFR estimates might imply the need for a smaller $\alpha_{\mathrm{CO}}$ conversion factor than the Galactic value, which we use in this work to allow a homogeneous comparison. A value $\alpha_{\mathrm{CO}} \simeq 1 M_{\odot}\left(\mathrm{K} \mathrm{km} \mathrm{s}^{-1} \mathrm{pc}^{2}\right)^{-1}$ is indeed usually adopted for (ultra-)luminous infrared galaxies (see, e.g., Bolatto et al. 2013, for a review). Assuming a lower $\alpha_{\mathrm{CO}}$ than the Galactic value would imply even lower $M\left(\mathrm{H}_{2}\right) / M_{\star}$ values for our two targets, increasing the observed tension with respect to the MS values. Alternatively, the BCG emission is contaminated by an AGN, which might result in biased-high SFRs. In the following we consider the 11 BCGs separately.

As discussed in Sect. 2 our IRAM $30 \mathrm{~m}$ targets are both radio galaxies; this is also the case for the other five $z \simeq(0.4-2.6)$ sources we observed in CO with the IRAM $30 \mathrm{~m}$ telescope (Castignani et al. 2019). MRC 1138-262 and the SpARCS1049+ $56 \mathrm{BCG}$ are powerful radio sources. They have $L_{4.5 \mathrm{GHz}}=1.9 \times$ $10^{28} \mathrm{~W} \mathrm{~Hz}^{-1}$ (e.g., Carilli et al. 1997; Pentericci et al. 1997; Miley et al. 2006) and $L_{1.4 \mathrm{GHz}}=4.2 \times 10^{24} \mathrm{~W} \mathrm{~Hz}^{-1}$ (Trudeau et al. 2019), respectively. Gobat et al. (2011) discussed the possibility that some obscured AGN activity is associated with the triplet of galaxies corresponding to the ClJ1449+0856 (proto)BCG, resulting in the observed $24 \mu \mathrm{m}$ emission. Candels-5001 at $z=3.47$ has an X-ray (2-10 keV) luminosity of $10^{42.5} \mathrm{erg} \mathrm{s}^{-1}$ which Fiore et al. (2012) attributed to stellar sources. However the authors also report for the source a $1.4 \mathrm{GHz}$ radio flux of $(15.3 \pm 6.3) \mu \mathrm{Jy}$, which implies a rest-frame $1.4 \mathrm{GHz}$ luminosity density $L_{1.4 \mathrm{GHz}} \simeq 1.2 \times 10^{24} \mathrm{~W} \mathrm{~Hz}^{-1}$, assuming a spectral index $\alpha=0.8$ (e.g., Chiaberge et al. 2009).

Although further multiwavelength observations of the BCGs are needed to have a robust evaluation of the AGN contamination, we note that for distant star-forming galaxies it is typically $20 \%$ at mid- to far-infrared wavelengths (e.g., Pozzi et al. 2012) and may be higher for active BCGs. However, Webb et al. (2015b) corrected the SFR of the SpARCS1049+56 BCG for a limited AGN contamination of $\sim 20 \%$. Furthermore, spectral energy distribution (SED) modeling of the five $z \simeq(0.4-2.6)$ sources of our IRAM $30 \mathrm{~m}$ campaign, selected within the COSMOS and DES SN deep fields, shows that the infrared to ultraviolet emission closely resembles that of ellipticals (Baldi et al. 2013; Castignani et al. 2019), more than that, steep-spectrum, typical of radio-loud quasars. The SEDs of these five BCGs are also consistent with those of other samples of radio loud star-forming galaxies in COSMOS (Delvecchio et al. 2017).

\subsection{Morphological classification of the BCGs}

Complex molecular gas morphologies and filamentary structures have been found associated with local gas-rich BCGs (e.g., Russell et al. 2016, 2017). Several observational studies have also proposed connections between the optical morphologies and the star formation properties of distant galaxies (van der Wel et al. 2014; Dimauro et al. 2018, 2019; Socolovsky et al. 2018, 2019; Puglisi et al. 2019; Freundlich et al. 2019). Inspired by these studies we investigate the morphological properties of the 11 BCGs considered in this work, while in Sect. 4.7 we discuss them in relation to their molecular gas reservoirs and star formation properties.

We looked for archival Hubble Space Telescope (HST) observations and found HST images for the ClJ1449+0856 ( $z=$ $1.99)$ and SpARCS1049+56 $(z=1.7)$ BCGs, MRC 1138-262 $(z=2.2)$, and 3C 244.1. We did not find any archival HST image for the other IRAM $30 \mathrm{~m}$ target SDSS J161112.65+550823.5. In Castignani et al. (2019) we present a morphological analysis of COSMOS-FRI 16, 31, and 70, based on archival HST ACS $F 814 W$ (I-band) images with a pixel scale of 0.03 arcsec. By running GALFIT (Peng et al. 2002, 2010) on these images we estimated half-light radii $r_{\mathrm{e}}=(4.9 \pm 0.8),(4.7 \pm 0.8)$, and $(2.9 \pm 0.8) \mathrm{kpc}$ for the three COSMOS-FRIs.

For the other four BCG candidates several archival HST images have been found. When there were multiple observations we visually inspected the images and chose those of higher resolution and better quality, as outlined in the following. We considered the archival WFPC2 image (F785LP filter) at a resolution of 0.1 arcsec for 3C244.1; WFC3 images for both C1J1449+0856 (filter $F 140 W$ ) and SpARCS1049+56 (filter $F 160 W$ ) BCGs, at a resolution of 0.128 arcsec; and the ACS/WFC image ( $F 814 W$ filter) for MRC 1138-262, at a resolution of 0.05 arcsec.

We then ran GALFIT by fitting the HST images of the BCGs with a Sèrsic law, as reported in Eq. (3) of Castignani et al. (2019). The fits were performed using a generic Tiny Tim (Krist 1995; Krist et al. 2011) point spread function for the different HST filters and cameras. Our GALFIT analysis yielded $r_{\mathrm{e}}=(12.6 \pm 0.8) \mathrm{kpc}$ for $3 \mathrm{C} 244.1, r_{\mathrm{e}}=(8.0 \pm 1.1) \mathrm{kpc}$ for the SpARCS1049+56 BCG, and $r_{\mathrm{e}}=(3.3 \pm 0.6) \mathrm{kpc}$ for MRC $1138-$ 262. For the $\mathrm{ClJ} 1449+0856 \mathrm{BCG}$ at $z \simeq 2$ several components are identified in the HST images by previous studies (Gobat et al. 2011; Strazzullo et al. 2018), which suggests that the BCG is still assembling and associated with a triplet of galaxies in a likely merging phase. Similarly to these studies, we denoted the three HST components as H1, H4, and H5. Our GALFIT analysis yielded $r_{\mathrm{e}}=(24.3 \pm 2.3) \mathrm{kpc}$ for $\mathrm{H} 1, r_{\mathrm{e}}=(9.5 \pm 1.1) \mathrm{kpc}$ for $\mathrm{H} 5$, and $r_{\mathrm{e}}<3.3 \mathrm{kpc}$ for $\mathrm{H} 4$. For $\mathrm{H} 4$ we report only a $3 \sigma$ upper limit because the $r_{\mathrm{e}}$ estimate is on the order of the pixel size (i.e., $\sim 1 \mathrm{kpc}$ at the redshift of the source).

At variance with our recent Castignani et al. (2019) study on COSMOS-FRI sources, our attempt to derive a robust 

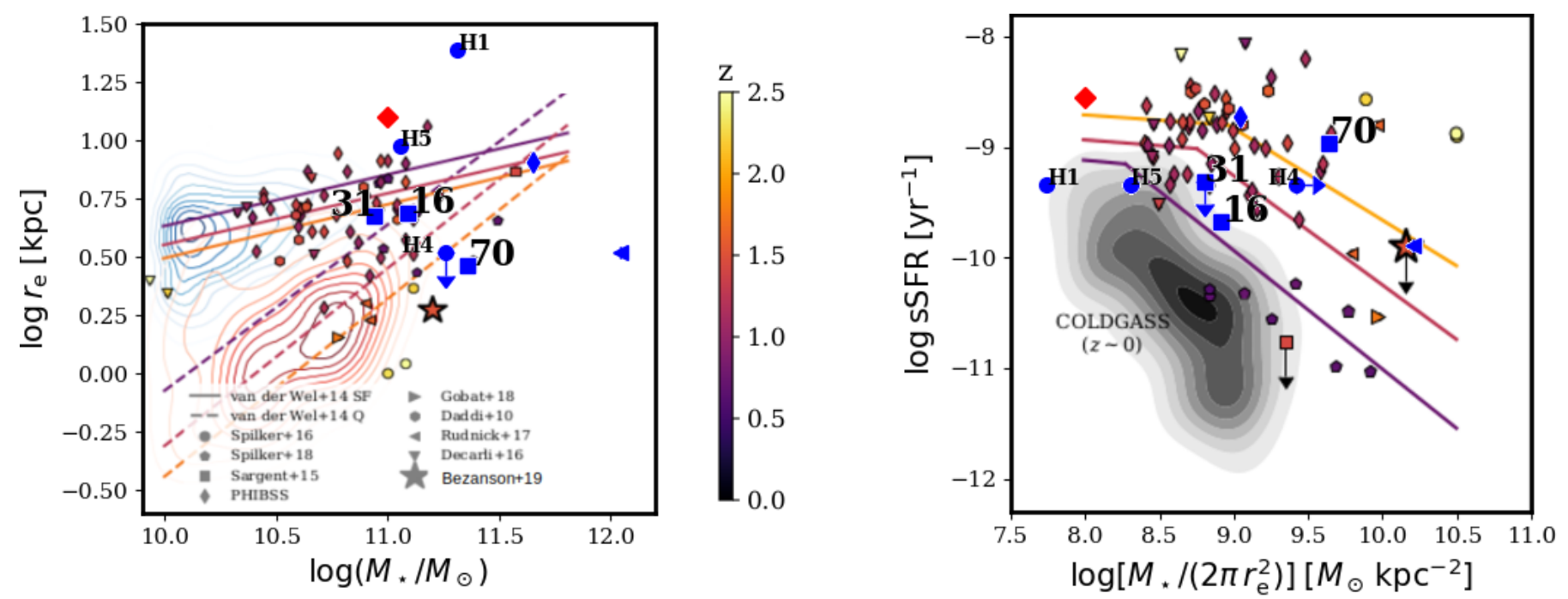

Fig. 4. Effective radius $\left(r_{\mathrm{e}}\right)$ vs. stellar mass (left) and specific SFR vs. stellar mass surface density (right). The red diamond is $3 \mathrm{C} 244.1$, while blue symbols correspond to ClJ1449+0856 (H1, H4, H5) and SpARCS1049+56 BCGs, MRC 1138-262, as well as COSMOS-FRI 16, 31, and 70, which are highlighted with the corresponding ID numbers. We refer to Fig. 2 for the legend. Comparison data points, contours, and empirical relations shown in Figs. 3 and 5 of Bezanson et al. (2019) are superimposed. See text for details.

Sèrsic index for the BCGs did not produce reliable results. This is mainly due to the presence of several substructures for the distant Spider Web galaxy at $z \simeq 2.2$, while for the other sources the resolution of $\sim 0.1$ arcsec was not high enough for robust estimates of the Sèrsic index.

\subsection{Compactness, star formation, and gas depletion}

The majority of the 11 considered BCGs tend to have low $M\left(\mathrm{H}_{2}\right) / M_{\star}$ values and low depletion timescales $\tau_{\text {dep }}$ compared to MS galaxies and distant cluster galaxies observed in $\mathrm{CO}$ (see Sect. 4.4). In particular, our two target BCGs 3C 244.1 and SDSS J161112.65+550823.5 have low ratios of molecular gas to stellar mass $M\left(\mathrm{H}_{2}\right) / M_{\star} \lesssim 0.2$, which suggest that gas depletion in these BCGs, and in the majority of the 11 considered BCGs, was effective. Similarly, low ratios $(\$ 10 \%$, Sargent et al. 2015; Gobat et al. 2018; Bezanson et al. 2019) have recently been found in distant ellipticals. These results motivated us to use the morphological analysis outlined in the previous section for the $11 \mathrm{BCG}$ to better understand the mechanisms governing their star formation fueling.

Similarly to Bezanson et al. (2019), in Fig. 4 we show the effective radius $\left(r_{\mathrm{e}}\right)$ versus the stellar mass (left) and the sSFR versus the stellar mass surface density (right), defined as $\Sigma_{\star}=$ $M_{\star} /\left(2 \pi r_{\mathrm{e}}^{2}\right)$. We report several star-forming and quiescent galaxies. We overplot in both panels of the figure 7 of the 11 distant BCGs that have $r_{\mathrm{e}}$ estimates, as described in Sect. 4.6. For the $\mathrm{ClJ} 1449+0856$ BCG we assume that the H1, H4, and H5 components have the same sSFR and contribute to the total BCG stellar mass of $M_{\star}=(5.0 \pm 2.7) \times 10^{11} M_{\odot}$ (Gobat et al. 2011; Castignani et al. 2019) proportionally to their HST $F 140 W$ WFC3 flux.

Comparison data points, contours, and empirical relations shown in Figs. 3 and 5 of Bezanson et al. (2019) are superimposed onto our Fig. 4 as follows. In the left panel, blue and red contours show respectively the location of star-forming and quiescent galaxies at $1<z<2$ in the 3D-HST survey (Brammer et al. 2011; Skelton et al. 2014; van der Wel et al. 2012). van der Wel et al. (2014) size-mass relations at different epochs are shown as solid and dashed lines for star-forming and quiescent galaxies, respectively. In the right panel, gray contours correspond to $z \sim 0$ galaxies from the COLDGASS survey (Saintonge et al. 2011). The solid lines show the broken power-law relations at $z=0.75$, 1.25, and 1.75 from Whitaker et al. (2017). In both panels the different colors of the comparison data points and lines correspond to different redshifts, as shown in the color bar in the left panel. In both panels galaxies from several samples with molecular gas and rest-frame optical size measurements are indicated by colored symbols (Daddi et al. 2010; Tacconi et al. 2010; Sargent et al. 2015; Decarli et al. 2016; Spilker et al. 2016, 2018; Rudnick et al. 2017; Gobat et al. 2018; Bezanson et al. 2019).

As shown in the left panel of the Figure, MRC 1138-262 and COSMOS-FRI 16, 31, and 70 have effective radii that are smaller than those found for star-forming MS galaxies of similar mass and redshift using the van der Wel et al. (2014) scaling relation (see also Castignani et al. 2019, for further discussion). These four sources are also those where high-resolution HST images with pixel sizes $\sim(0.03-0.05)$ arcsec are available. On the other hand, for 3C 244.1 and both CIJ1449+0856 and SpARCS1049+56 BCGs lower resolution images (pixel size $\sim 0.1$ arcsec) have been used to estimate $r_{\mathrm{e}}$. These sources have estimated effective radii that are consistent with those of MS field galaxies. It might be possible that the poorer resolution resulted in biased-high $r_{\mathrm{e}}$ estimates for these three sources.

Furthermore, the ClJ1449+0856 BCG is a triplet of possibly interacting sources, among them $\mathrm{H} 4$ has a small estimated size $r_{\mathrm{e}}<3.3 \mathrm{kpc}$. As discussed in Strazzullo et al. (2018), the origin of the $\mathrm{CO}$ emission associated with the (proto-)BCG could be an additional star-forming $\sim 300 M_{\odot} \mathrm{yr}^{-1}$ component of similar size as H4. These aspects show that the ClJ1449+0856 BCG system is complex, while the morphological analysis does not exclude the presence of compact star-forming components. Similarly, MRC 1138-262 shows several clumpy substructures likely associated with stellar components, found in the archival HST ACS images ( $F 475 W$ and $F 814 W$ filters); see also Sect. 4.6.

Although the small sample size and the large scatter in the data points prevent us from drawing firm conclusions, our morphological analysis reveals that five out of the seven considered BCGs (i.e., $71 \% \pm 17 \%$ ) either have smaller $r_{\mathrm{e}}$ than those of 
MS field star-forming galaxies or show a complex morphology with several likely star-forming components. For the remaining two BCGs (3C 244.1 and the SpARCS1049+56 BCG), our GALFIT analysis did not show a compact morphology. However, it might be that this is due to the lower resolution images (pixel size $\sim 0.1$ arcsec) used for the analysis.

As a comparison, recent work by Ito et al. (2019) shows instead that distant $z \sim 4$ proto-BCGs have sizes, on average, that are $\sim 28 \%$ larger than those of field galaxies for a fixed brightness. We suggest that the apparent discrepancy with respect to our results is ultimately due to the different sample selection. Increasing the sample of distant BCGs and CO observations will help to more accurately characterize the population of high- $z$ BCGs.

Figure 4 (right) shows that the majority of the considered BCGs have a high sSFR and a stellar mass surface density $\Sigma_{\star}$ that is consistent with those of distant star-forming galaxies from the literature. However, COSMOS FRI 70 and MRC 1138-262 have a higher $\Sigma_{\star}$ than the other distant BCGs, which is consistent with the fact that they are also more compact. As can be seen in the figure, high values $\left(\log \left(\Sigma_{\star} /\left(M_{\odot} \mathrm{kpc}^{-2}\right)\right) \sim 9.5-10\right)$ have been also found for other compact sources from the literature (Spilker et al. 2016; Rudnick et al. 2017; Bezanson et al. 2019).

Interestingly, MRC 1138-262 has both $\Sigma_{\star}$ and sSFR values that are very similar to those reported by Bezanson et al. (2019) for their quiescent compact elliptical at $z=1.5$. However, we note that the SFR of MRC 1138-262 is uncertain and its sSFR might by even a factor of $\sim 10$ higher than reported in Fig. 4. For MRC 1138-262 we assume SFR $=142 M_{\odot} \mathrm{yr}^{-1}$ (Hatch et al. 2008; Emonts et al. 2016), with large uncertainties (see Figs. 2 and 3) since an SFR of up to $\sim 1400 M_{\odot} \mathrm{yr}^{-1}$ (Seymour et al. 2012) has been reported for the BCG.

\subsection{Compactness and quenching}

The relatively low depletion times combined with the compactness associated with the distant BCGs are reminiscent of the compaction phase suggested for $z \sim 2-4$ galaxies by observations (e.g., Barro et al. 2013, 2017) and by simulations (Zolotov et al. 2015; Tacchella et al. 2016a,b) in which massive star-forming galaxies experience an enhancement of the star formation due to gas deposition at their centers, before an inside-out gas depletion and then followed by quenching.

We compare our results with those independently found for different samples of distant galaxies. Recent studies by Socolovsky et al. $(2018,2019)$ show that galaxies with high $\mathrm{sSFR} \simeq 1 \mathrm{Gyr}^{-1}$ in dense environments at $0.5<z<1.0$ are strongly depleted due to rapid environmental quenching, and are likely to evolve into post-starbursts (PSBs). They also tend to have larger effective radii than those in the field, possibly because the most compact star-forming galaxies are preferentially quenched in dense environments.

These findings seem to be in apparent disagreement with the existence of compact distant star-forming BCGs (Sect. 4.7). The discrepancy can be possibly explained by noting that relatively compact sources such as the Spider Web galaxies and COSMOS-FRI 16, 31, and 70 represent a rare population of BCGs observed during a significant star formation activity. The BCGs will deplete their molecular gas in a relatively short time and then evolve into quenched compact ellipticals, as the one reported in the recent study by Bezanson et al. (2019). This picture is also in agreement with recent findings by Puglisi et al. (2019), who studied a sample of star-forming galaxies at $1.1 \leq z \leq 1.7$ and found a non-negligible fraction $>29 \%$ of compact sources possibly observed as early PSBs.

\section{Summary and conclusions}

We have investigated the effect of dense Mpc-scale environments in processing molecular gas of distant star-forming brightest cluster galaxies (BCGs) with the final goal of better understanding the processes involved in the BCG growth and the regulation of star formation in distant BCGs. To this end we observed in CO with the IRAM $30 \mathrm{~m}$ telescope two star-forming distant BCGs: 3C 244.1 $(z=0.4)$ and SDSS J161112.65+550823.5 $(z=$ $0.9)$ with $\mathrm{SFR}=281 M_{\odot} \mathrm{yr}^{-1}$ and $766 M_{\odot} \mathrm{yr}^{-1}$, respectively, as inferred from $24 \mu \mathrm{m}$ Spitzer-MIPS fluxes (Webb et al. 2015a). By adopting standard CO-to- $\mathrm{H}_{2}$ conversion factors we set robust upper limits to their ratio of molecular gas to stellar mass $M\left(\mathrm{H}_{2}\right) / M_{\star} \lesssim 0.2$ and depletion time $\tau_{\text {dep }} \lesssim 40$ Myr.

We then compared these results with those found for a compilation of $\sim 100$ distant cluster galaxies with $\mathrm{CO}$ observations and stellar mass estimates from the literature, which include nine additional BCG candidates at $z \sim 0.4-3.5$, five of them are from our recent work (Castignani et al. 2019) on distant radio galaxies in dense megaparsec-scale environments. This comparison places the two targeted BCGs among the distant cluster galaxies with the lowest gas fractions and shortest depletion times. More in general, by considering the sample of 11 BCGs with $\mathrm{CO}$ observations we found that these rare star-forming BCGs, given the large uncertainties and scatter in the data points, have SFRs and $M\left(\mathrm{H}_{2}\right) / M_{\star}$ that are generally consistent with the values found using empirical relations for the MS (Tacconi et al. 2018; Speagle et al. 2014) and with those of the other distant cluster galaxies observed in $\mathrm{CO}$. Nevertheless, the majority (i.e., $64 \% \pm 15 \%$ and $73 \% \pm 18 \%$ ) of the 11 BCGs have lower $M\left(\mathrm{H}_{2}\right) / M_{\star}$ values and lower $\tau_{\mathrm{dep}}$, respectively, than those estimated for MS galaxies of similar stellar mass and redshift. Similarly, the statistical analysis based on the Kolmogorov-Smirnov test tentatively suggests that the values of $M\left(\mathrm{H}_{2}\right) / M_{\star}$ and $\tau_{\text {dep }}$ for the 11 BCGs deviates, with a significance of $\sim 2 \sigma$, from those of the cluster galaxies from the comparison sample.

These results favor a scenario where star-forming BCGs assemble a significant fraction $f=\tau_{\text {dep }} \mathrm{SFR} / M_{\star} \simeq 16 \%$ of their stellar mass within a timescale $\tau_{\text {dep }} \sim(0.04-7)$ Gyr, not only during early stages $(z \gtrsim 2)$, but also at later $(z \lesssim 1)$ epochs, consistent with previous work (Lidman et al. 2012; McDonald et al. 2014). These findings seem to favor the presence of environmental mechanisms (e.g., strangulation, ram pressure stripping, and galaxy harassment) that might prevent the replenishment of gas feeding the star formation, while in the meantime allowing the exhaustion of the BCG gas reservoirs on a relatively short timescale $\sim \tau_{\text {dep }}$, to sustain the observed star formation. To further explore this scenario we investigated the possibility that the compactness may help the regulation of the star formation in distant BCGs.

A morphological analysis using GALFIT was done for 7 of the 11 BCGs. The seven sources, including our IRAM $30 \mathrm{~m}$ target 3C 244.1, have archival HST observations. We found that $71 \% \pm 17 \%$ of the BCGs are compact or show star-forming components or substructures. The two most compact BCGs, which are also the most distant, in the subsample of seven also have high stellar mass surface density $\log \left(\Sigma_{\star} /\left(M_{\odot} \mathrm{kpc}^{-2}\right)\right) \sim 9.5-10$, similar to that previously found for other distant compact sources (Spilker et al. 2016; Rudnick et al. 2017; Bezanson et al. 2019).

We speculate that, for a significant fraction of distant starforming BCGs, compact morphologies and star-forming components may both favor the rapid exhaustion of molecular gas and ultimately help to quench the BCGs. Higher resolution and higher surface brightness sensitivity observations of 
distant BCGs will help to distinguish between different gasprocessing mechanisms (e.g., galaxy harassment, strangulation, ram pressure stripping, or tidal stripping), possibly responsible for quenching the galaxies. The BCGs considered in this work are excellent targets for ALMA as well as next-generation telescopes such as the James Webb Space Telescope.

Acknowledgements. We thank the anonymous referee for helpful comments which contributed to improve the paper significantly. GC acknowledges financial support from the Swiss National Science Foundation (SNSF). This work is based on observations carried out under project number 065-18 with the IRAM $30 \mathrm{~m}$ telescope. IRAM is supported by INSU/CNRS (France), MPG (Germany) and IGN (Spain). This publications has made use of data products from the NASA/IPAC Extragalactic Database (NED).

\section{References}

Alberts, S., Pope, A., Brodwin, M., et al. 2016, ApJ, 825, 72 Aravena, M., Carilli, C. L., Salvato, M., et al. 2012, MNRAS, 426, 258 Bai, L., Rieke, G. H., Rieke, M. J., et al. 2009, ApJ, 693, 1840 Baldi, T., Chiaberge, M., Capetti, A., et al. 2013, ApJ, 762, 30 Barro, G., Faber, S. M., Pérez-González, P. G., et al. 2013, ApJ, 765, 104 Barro, G., Faber, S. M., Koo, D. C., et al. 2017, ApJ, 840, 47 Bell, E. F., McIntosh, D. H., Katz, N., et al. 2003, ApJS, 149, 289 Bezanson, R., Spilker, J., Williams, C. C., et al. 2019, ApJ, 873, 19 Bigiel, F., Leroy, A., Walter, F., et al. 2008, AJ, 136, 2846

Bleem, L. E., Stalder, B., de Haan, T., et al. 2015, ApJS, 216, 27 Bolatto, A. D., Mark, W., \& Adam, K. 2013, ARA\&A, 51, 207 Bonaventura, N. R., Webb, T. M. A., Muzzin, A., et al. 2017, MNRAS, 469, 1259

Bothwell, M. S., Smail, I., Chapman, S. C., et al. 2013, MNRAS, 429, 3047 Brammer, G. B., Whitaker, K. E., van Dokkum, P. G., et al. 2011, ApJ, 739, 24 Brodwin, M., Stanford, S. A., Gonzalez, A. H., et al. 2013, ApJ, 779, 138 Bruzual, G., \& Charlot, S. 2003, MNRAS, 344, 1000

Carilli, C. L., \& Walter, F. 2013, ARA\&A, 51, 105

Carilli, C. L., Röttgering, H. J. A., van Ojik, R., et al. 1997, ApJS, 109, 1 Carilli, C. L., Daddi, E., Riechers, D., et al. 2010, ApJ, 714, 1407 Castignani, G., Chiaberge, M., Celotti, A., et al. 2014, ApJ, 792, 114 Castignani, G., Combes, F., Salomé, P., et al. 2018, A\&A, 617, A103 Castignani, G., Combes, F., Salomé, P., et al. 2019, A\&A, 623, A48 Chary, R., \& Elbaz, D. 2001, ApJ, 556, 562

Chiaberge, M., Tremblay, G., Capetti, A., et al. 2009, ApJ, 696, 1103 Chung, S. M., Gonzalez, A. H., Clowe, D., et al. 2010, ApJ, 725, 153 Collins, C. A., Stott, J. P., Hilton, M., et al. 2009, Nature, 458, 603 Coogan, R. T., Daddi, E., Sargent, M. T., et al. 2018, MNRAS, 479, 703 Cooke, E. A., Hatch, N. A., Stern, D., et al. 2016, ApJ, 816, 83 Cybulski, R., Yun, M. S., Erickson, N., et al. 2016, MNRAS, 459, 3287 Daddi, E., Bournaud, F., Walter, F., et al. 2010, ApJ, 713, 686 Daddi, E., Dannerbauer, H., Liu, D., et al. 2015, A\&A, 577, A46 Dannerbauer, H., Lehnert, M. D., Emonts, B., et al. 2017, A\&A, 608, A48 Decarli, R., Walter, F., Aravena, M., et al. 2016, ApJ, 833, 70 Dekel, A., Birnboim, Y., Engel, G., et al. 2009a, Nature, 457, 451 Dekel, A., Sari, R., Ceverino, D., et al. 2009b, ApJ, 703, 785 De Lucia, G., \& Blaizot, J. 2007, MNRAS, 375, 2

Delvecchio, I., Smolcic, V., Zamorani, G., et al. 2017, A\&A, 602, A3 Demarco, R., Wilson, G., Muzzin, A., et al. 2010, ApJ, 711, 1185

Dimauro, P., Huertas-Company, M., Daddi, E., et al. 2018, MNRAS, 478, 5410 Dimauro, P., Huertas-Company, M., Daddi, E., et al. 2019, MNRAS, 489, 4135 Dressler, A. 1980, ApJ, 236, 351

Edge, A. C. 2001, MNRAS, 328, 762

Emonts, B. H. C., Feain, I., Röttgering, H. J. A., et al. 2013, MNRAS, 430, 3465 Emonts, B. H. C., Lehnert, M. D., Villar-Martín, M., et al. 2016, Science, 354, 1128

Fanaroff, B. L., \& Riley, J. M. 1974, MNRAS, 167, 31P

Fiore, F., Puccetti, S., Grazian, A., et al. 2012, A\&A, 537, A16

Fogarty, K., Postman, M., Li, Y., et al. 2019, ApJ, 879, 103

Fraser-McKelvie, A., Brown, M. J. I., \& Pimbblet, K. A. 2014, MNRAS, 444, 63

Freundlich, J., Combes, F., Tacconi, L. J., et al. 2019, A\&A, 622, A105 Geach, J. E., Smail, I., Moran, S. M., et al. 2011, ApJ, 730, L19 Ginolfi, M., Maiolino, R., Nagao, T., et al. 2017, MNRAS, 468, 3468 Gobat, R., Daddi, E., Onodera, M., et al. 2011, A\&A, 526, A133 Gobat, R., Daddi, E., Magdis, G., et al. 2018, Nat. Astron., 2, 239 Gómez-Guijarro, C., Riechers, D. A., Pavesi, R., et al. 2019, ApJ, 872, 117 Gunn, J. E., \& Gott, III., J. R. 1972, ApJ, 176, 1
Hamer, S. L., Edge, A. C., Swinbank, A. M., et al. 2012, MNRAS, 421, 3409 Hatch, N. A., Overzier, R. A., Röttgering, H. J. A., et al. 2008, MNRAS, 383, 931

Hatch, N. A., Overzier, R. A., Kurk, J. D., et al. 2009, MNRAS, 395, 114 Hausman, M. A., \& Ostriker, J. P. 1978, ApJ, 224, 320

Hayashi, M., Tadaki, K., Kodama, T., et al. 2018, ApJ, 856, 118 Hill, G. J., \& Lilly, S. J. 1991, ApJ, 367, 1

Ito, K., Kashikawa, N., Toshikawa, J., et al. 2019, ApJ, 878, 68 Ivison, R. J., Swinbank, A. M., Smail, I., et al. 2013, ApJ, 772, 137 Jablonka, P., Combes, F., Rines, K., et al. 2013, A\&A, 557, A103

Kramer, C., Peñalver, J., \& Greve, A. 2013, Improvement of the IRAM 30m Telescope Beam Pattern, www.iram-institute.org/medias/uploads/ eb2013-v8.2.pdf

Kennicutt, R. C. J. 1998, ARA\&A, 36, 189

Kneissl, R., del Carmen Polletta, M., Martinache, C., et al. 2019, A\&A, 625, A96

Kodama, T., Smail, I., Nakata, F., et al. 2001, ApJ, 562, 9

Koyama, Y., Kodama, T., Tadaki, K., et al. 2014, ApJ, 789, 18

Krist, J. 1995, ASP Conf. Ser., 77, 349

Krist, J. E., Hook, R. N., \& Stoehr, F. 2011, Proc. SPIE, 8127

Larson, R. B., Tinsley, B. M., Caldwell, C. N., et al. 1980, ApJ, 237, 69

Lauer, T. R., Postman, M., Strauss, M. A., et al. 2014, ApJ, 797, 82

Lee, M. M., Tanaka, I., Kawabe, R., et al. 2017, ApJ, 842, 55

Leroy, A. K., Walter, F., Sandstrom, K, et al. 2013, AJ, 146, 19

Lidman, C., Suherli, J., Muzzin, A., et al. 2012, MNRAS, 427, 550

Madau, P., \& Dickinson, M. 2014, ARA\&A, 52, 415

McDonald, M., Benson, B., Veilleux, S., et al. 2013, ApJ, 765, L37

McDonald, M., Swinbank, M., Edge, A. C., et al. 2014, ApJ, 784, 18

McDonald, M., Stalder, B., Bayliss, M., et al. 2016, ApJ, 817, 86

McNamara, B. R., Russell, H. R., Nulsen, P. E. J., et al. 2014, ApJ, 785, 44

Miley, G. K., Overzier, R. A., Zirm, A. W., et al. 2006, ApJ, 650, 29

Moore, B., Lake, G., Quinn, T., et al. 1999, MNRAS, 304, 465

Moravec, E., Gonzalez, A. H., Stern, D., et al. 2019, ApJ, 871, 186

Muzzin, A., Wilson, G., \& Yee, H. K. C. 2009, ApJ, 698, 1934

Muzzin, A., Wilson, G., \& Yee, H. K. C. 2012, ApJ, 746, 188

Newman, A. B., Ellis, R. S., Andreon, S., et al. 2014, ApJ, 788, 51

Noble, A. G., McDonald, M., Muzzin, A., et al. 2017, ApJ, 842, 21

Noble, A. G., Muzzin, A., McDonald, M., et al. 2019, ApJ, 870, 56

Ocvirk, P., Pichon, C., Teyssier, R., et al. 2008, MNRAS, 390, 1326

Olivares, V., Salomé, P., Combes, F., et al. 2019, A\&A, 631, A22

Ostriker, J. P., \& Tremaine, S. D. 1975, ApJ, 202, 113

Papadopoulos, P. P., Röttgering, H. J. A., van der Werf, P. P., et al. 2000, ApJ, 528, 626

Peng, C. Y., Ho, L. C., Impey, C. D., et al. 2002, AJ, 124, 266

Peng, Y.-J., Lilly, S. J., Kovač, K., et al. 2010, ApJ, 721, 193

Pentericci, L., Röttgering, H. J. A., Miley, G. K., et al. 1997, A\&A, 326, 580

Planck Collaboration VI. 2018, A\&A, submitted [arXiv:1807.06209]

Pozzi, F., Vignali, C., Gruppioni, C., et al. 2012, MNRAS, 423, 1909

Puglisi, A., Daddi, E., Liu, D., et al. 2019, ApJ, 877, 23

Riechers, D. A., Capak, P. L., Carilli, C. L., et al. 2010, ApJ, 720, 131

Riess, A. G., Casertano, S., Yuan, W., et al. 2019, ApJ, 876, 85

Roediger, J. C., \& Courteau, S. 2015, MNRAS, 452, 3209

Rudnick, G., Hodge, J., Walter, F., et al. 2017, ApJ, 849, 27

Russell, H. R., McNamara, B. R., Edge, A. C., et al. 2014, ApJ, 784, 78

Russell, H. R., McNamara, B. R., Fabian, A. C., et al. 2016, MNRAS, 458, 3134 Russell, H. R., McDonald, M., McNamara, B. R., et al. 2017, ApJ, 836, 130 Russell, H. R., McNamara, B. R., Fabian, A. C., et al. 2019, MNRAS, 490, 3025 Saintonge, A., Kauffmann, G., Kramer, C., et al. 2011, MNRAS, 415, 32 Salomé, P., \& Combes, F. 2003, A\&A, 412, 657

Salomé, P., Combes, F., Edge, A. C., et al. 2006, A\&A, 454, 437

Santos, J. S., Altieri, B., Valtchanov, I., et al. 2015, MNRAS, 447, 65 Sargent, M. T., Daddi, E., Bournaud, F., et al. 2015, ApJ, 806, L20

Schruba, A., Leroy, A. K., Walter, F., et al. 2011, AJ, 142, 37

Serjeant, S., \& Marchetti, L. 2014, MNRAS, 443, 3118

Seymour, N., Altieri, B., De Breuck, C., et al. 2012, ApJ, 755, 146

Skelton, R. E., Whitaker, K. E., Momcheva, I. G., et al. 2014, ApJS, 214, 24

Smith, G. P., Haines, C. P., Pereira, M. J., et al. 2010, A\&A, 518, A1

Socolovsky, M., Almaini, O., Hatch, N. A., et al. 2018, MNRAS, 476, 1242

Socolovsky, M., Maltby, D. T., Hatch, N. A., et al. 2019, MNRAS, 482, 1640

Solomon, P. M., \& Vanden Bout, P. A. 2005, ARA\&A, 43, 677

Solomon, P. M., Downes, D., Radford, S. J. E., et al. 1997, ApJ, 478, 144

Speagle, J. S., Steinhardt, C. L., Capak, P. L., et al. 2014, ApJS, 214, 15

Spilker, J. S., Bezanson, R., Marrone, D. P., et al. 2016, ApJ, 832, 19

Spilker, J., Bezanson, R., Barišić, I., et al. 2018, ApJ, 860, 103

Strazzullo, V., Daddi, E., Gobat, R., et al. 2016, ApJ, 833, 20

Strazzullo, V., Coogan, R. T., Daddi, E., et al. 2018, ApJ, 862, 64

Strazzullo, V., Strazzullo, V., Pannella, M., Mohr, J. J., et al. 2019, A\&A, 622, A117 
Stott, J. P., Collins, C. A., Burke, C., et al. 2011, MNRAS, 414, 445 Stott, J. P., Hickox, R. C., Edge, A. C., et al. 2012, MNRAS, 422, 2213 Tacchella, S., Dekel, A., Carollo, C. M., et al. 2016a, MNRAS, 457, 2790 Tacchella, S., Dekel, A., Carollo, C. M., et al. 2016b, MNRAS, 458, 242 Tacconi, L. J., Genzel, R., Neri, R., et al. 2010, Nature, 463, 781 Tacconi, L. J., Genzel, R., Saintonge, A., et al. 2018, ApJ, 853, 179 Tadaki, K., Kodama, T., Ota, K., et al. 2012, MNRAS, 423, 2617

Tadaki, K., Kodama, T., Tamura, Y., et al. 2014, ApJ, 788, 23

Tadaki, K., Kodama, T., Hayashi, M., et al. 2019, PASJ, 71, 40

Tan, Q., Daddi, E., Magdis, G., et al. 2014, A\&A, 569, A98

Tanaka, M., Toft, S., Marchesini, D., et al. 2013, ApJ, 772, 113

Tran, K.-V. H., Papovich, C., Saintonge, A., et al. 2010, ApJ, 719, 126

Tremblay, G. R., Oonk, J. B. R., Combes, F., et al. 2016, Nature, 534, 218

Trudeau, A., Webb, T., Hlavacek-Larrondo, J., et al. 2019, MNRAS, 487, 1210

Umehata, H., Tamura, Y., Kohno, K., et al. 2015, ApJ, 815, 8

van der Wel, A., Bell, E. F., Häussler, B., et al. 2012, ApJS, 203, 24

van der Wel, A., Franx, M., van Dokkum, P. G., et al. 2014, ApJ, 788, 28

von der Linden, A., Best, P. N., Kauffmann, G., et al. 2007, MNRAS, 379, 867
Wagg, J., Pope, A., Alberts, S., et al. 2012, ApJ, 752, 91

Walter, F., Decarli, R., Carilli, C., et al. 2012, Nature, 486, 233

Wang, T., Elbaz, D., Daddi, E., et al. 2016, ApJ, 828, 56

Wang, T., Elbaz, D., Daddi, E., et al. 2018, ApJ, 867, 29

Webb, T. M. A., O’Donnell, D., Yee, H. K. C., et al. 2013, AJ, 146, 84

Webb, T. M. A., Muzzin, A., Noble, A., et al. 2015a, ApJ, 814, 96

Webb, T. M. A., Noble, A., DeGroot, A., et al. 2015b, ApJ, 809, 173

Webb, T. M. A., Lowenthal, J., Yun, M., et al. 2017, ApJ, 844, 17

Whitaker, K. E., Bezanson, R., van Dokkum, P. G., et al. 2017, ApJ, 838, 19

White, S. D. M. 1976, MNRAS, 177, 717

Wilson, G., Muzzin, A., \& Yee, H. K. C. 2009, ApJ, 698, 1943

Wright, A. H., Robotham, A. S. G., Driver, S. P., et al. 2017, MNRAS, 470, 283

Young, J. S., Xie, S., Tacconi, L., et al. 1995, ApJS, 98, 219

Yu, H., Tozzi, P., van Weeren, R., et al. 2018, ApJ, 853, 100

Zeimann, G. R., Stanford, S. A., Brodwin, M., et al. 2013, ApJ, 779, 137

Zhang, Y., Miller, C., McKay, T., et al. 2016, ApJ, 816, 98

Zirbel, E. L. 1996, ApJ, 473, 713

Zolotov, A., Dekel, A., Mandelker, N., et al. 2015, MNRAS, 450, 2327 


\section{Appendix A: Compilation of distant cluster galaxies}

Table A.1. Properties of distant $z \gtrsim 0.2$ cluster galaxies observed in CO.

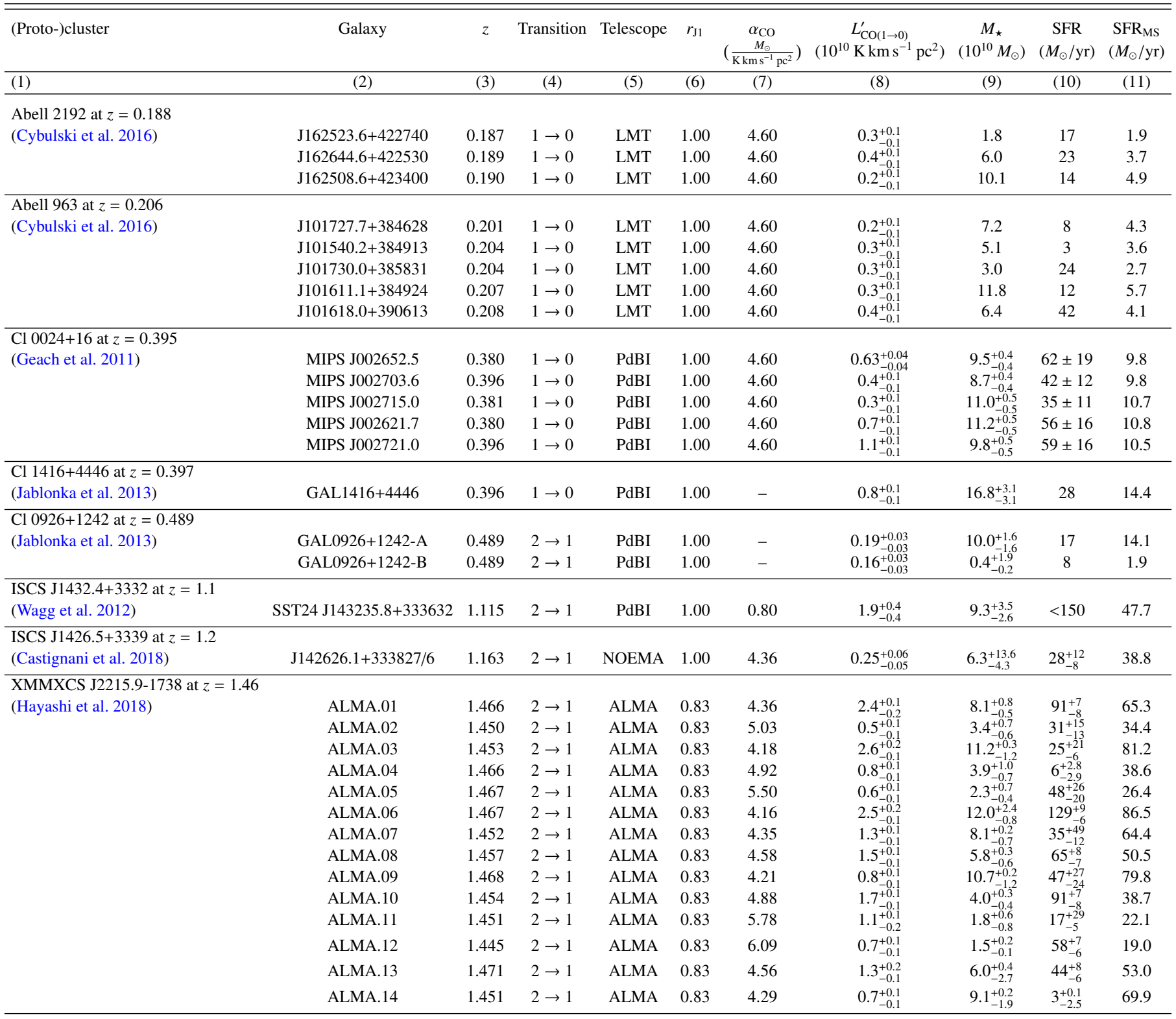

Notes. Cybulski et al. (2016) report infrared luminosities for their $z \sim 0.2$ cluster galaxies, which we have converted into SFR estimates using the Kennicutt (1998) relation. Jablonka et al. (2013) did not assume any value of $\alpha_{\mathrm{CO}}$; these sources are flagged with "-" in the $\alpha_{\mathrm{CO}}$ column. In our previous work (Castignani et al. 2018) we detected in $\mathrm{CO}(2 \rightarrow 1)$ two unresolved cluster galaxies, with the same redshift and $M_{\star}$ (Zeimann et al. 2013), that we show in the table. Consistently with Castignani et al. (2018) we also report $L_{\mathrm{CO}(1 \rightarrow 0)}^{\prime}$ and the SFR derived from the $24 \mu \mathrm{m}$ observer frame flux, for each of two galaxies, assuming that they equally contribute to the observed (unresolved) emission. Consistently with the order of preference adopted by Hayashi et al. (2018), we report the SFR estimated using both UV and $24 \mu \mathrm{m}$ observer frame emission, when available (i.e., for the sources with ID ALMA.01, 06, 08, 10, 12, 13, and 15). We report the SFR estimated from the mid-IR to optical SED for the remaining galaxies with ID: ALMA.02, 03, 04, 05, 07, 09, 11, 14, 16, and 17). Kneissl et al. (2019) report gas masses assuming two separate values of $\alpha_{\mathrm{CO}}$, which are listed in this Table. For the Aravena et al. (2012) sources the reported SFRs are the average between those derived from infrared luminosity and from SED fitting. For the Coogan et al. (2018) Cl J1449+0856 galaxies with $M_{\star}$ estimates, reported in this table, we list the SFRs derived from the $870 \mu \mathrm{m}$ observer frame flux. In the cases where the sources are not detected in $\mathrm{CO}(1 \rightarrow 0)$, we have used the $\mathrm{CO}(4 \rightarrow 3)$ detections to estimate $L_{\mathrm{CO}(1 \rightarrow 0)}^{\prime}$, by assuming an excitation ratio $r_{41}=0.36$, equal to the mean between the ratios of the two sources (ID 6, B1) with both $\mathrm{CO}(1 \rightarrow 0)$ and $\mathrm{CO}(4 \rightarrow 3)$ detections. Coogan et al. (2018) did not report any value of $\alpha_{\mathrm{CO}}$ for their source S7; this source is flagged with "-" in the $\alpha_{\text {CO }}$ column. Wang et al. (2018) report infrared luminosities for their $z \sim 2.5$ cluster galaxies, which we have converted into SFR estimates using the Kennicutt (1998) relation. 
G. Castignani et al.: Molecular gas in distant brightest cluster galaxies

Table A.1. continued.

\begin{tabular}{|c|c|c|c|c|c|c|c|c|c|c|}
\hline (Proto-)cluster & Galaxy & $z$ & Transition & Telescope & $r_{\mathrm{J} 1}$ & $\begin{array}{c}\alpha_{\mathrm{CO}} \\
\left(\frac{M_{\odot}}{\mathrm{Kkm} \mathrm{s}^{-1} \mathrm{pc}^{2}}\right)\end{array}$ & $\begin{array}{c}L_{\mathrm{CO}(1 \rightarrow 0)}^{\prime} \\
\left(10^{10} \mathrm{~K} \mathrm{~km} \mathrm{~s}^{-1} \mathrm{pc}^{2}\right)\end{array}$ & $\begin{array}{c}M_{\star} \\
\left(10^{10} M_{\odot}\right)\end{array}$ & $\begin{array}{c}\text { SFR } \\
\left(M_{\odot} / \mathrm{yr}\right)\end{array}$ & $\begin{array}{l}\mathrm{SFR}_{\mathrm{MS}} \\
\left(M_{\odot} / \mathrm{yr}\right)\end{array}$ \\
\hline \multirow[t]{4}{*}{$(1)$} & (2) & (3) & (4) & (5) & (6) & (7) & (8) & (9) & (10) & (11) \\
\hline & ALMA.15 & 1.465 & $2 \rightarrow 1$ & ALMA & 0.83 & 4.98 & $1.3_{-0.2}^{+0.1}$ & $3.6_{-0.2}^{+1.7}$ & $62_{-9}^{+8}$ & 36.7 \\
\hline & ALMA.16 & 1.465 & $2 \rightarrow 1$ & ALMA & 0.83 & 5.15 & $1.7_{-0.2}^{+0.1}$ & $3.1_{-0.5}^{+0.3}$ & $37_{-11}^{+29}$ & 32.7 \\
\hline & ALMA.17 & 1.460 & $2 \rightarrow 1$ & ALMA & 0.83 & 5.41 & $1.1_{-0.1}^{+0.1}$ & $2.5_{-0.5}^{+1.8}$ & $123_{-80}^{+51}$ & 27.5 \\
\hline \multirow[t]{2}{*}{$\begin{array}{l}\text { PLCK G073.4-57.5 at } z=1.5 \\
\text { (Kneissl et al. 2019) }\end{array}$} & 3 & 1.543 & $5 \rightarrow 4$ & ALMA & 0.32 & $4.36,0.80$ & $4.6_{-0.9}^{+0.9}$ & $3.9_{-0.7}^{+5.7}$ & $109_{-12}^{+16}$ & 41.3 \\
\hline & 8 & 1.545 & $5 \rightarrow 4$ & ALMA & 0.32 & $4.36,0.80$ & $0.3_{-0.1}^{+0.1}$ & $11.5_{-2.4}^{+2.0}$ & $381_{-41}^{+48}$ & 90.2 \\
\hline \multirow[t]{2}{*}{$\begin{array}{l}\text { COSMOS cluster candidate at } z=1.55 \\
\text { (Aravena et al. 2012) }\end{array}$} & 51613 & 1.517 & $1 \rightarrow 0$ & JVLA & 1.00 & 3.60 & $2.4_{-0.6}^{+0.6}$ & $4.5_{-1.5}^{+1.8}$ & $114 \pm 17$ & 44.8 \\
\hline & 51858 & 1.556 & $1 \rightarrow 0$ & JVLA & 1.00 & 3.60 & $1.2_{-0.4}^{+0.4}$ & $6.0_{-1.0}^{+1.6}$ & $190 \pm 28$ & 57.1 \\
\hline \multirow[t]{8}{*}{$\begin{array}{l}\text { SpARCS J022546-035517 at } z=1.59 \\
\text { (Noble et al. 2019) }\end{array}$} & J0225-371 & 1.599 & $2 \rightarrow 1$ & ALMA & 0.77 & 4.36 & $5.3_{-0.4}^{+0.4}$ & $6.3_{-0.9}^{+0.8}$ & $173 \pm 76$ & 61.4 \\
\hline & J0225-460 & 1.600 & $2 \rightarrow 1$ & ALMA & 0.77 & 4.36 & $2.1_{-0.2}^{+0.2}$ & $9.1_{-3.5}^{+6.0}$ & $116 \pm 60$ & 80.2 \\
\hline & J0225-281 & 1.611 & $2 \rightarrow 1$ & ALMA & 0.77 & 4.36 & $3.4_{-0.3}^{+0.3}$ & $5.4_{-2.6}^{+3.5}$ & $120 \pm 50$ & 55.5 \\
\hline & J0225-541 & 1.611 & $2 \rightarrow 1$ & ALMA & 0.77 & 4.36 & $4.8_{-1.1}^{+1.1}$ & $6.6_{-0.9}^{+0.8}$ & $82 \pm 30$ & 64.1 \\
\hline & J0225-429 & 1.602 & $2 \rightarrow 1$ & ALMA & 0.77 & 4.36 & $1.1_{-0.2}^{+0.2}$ & $0.6_{-0.1}^{+1.9}$ & $178 \pm 83$ & 11.2 \\
\hline & J0225-407 & 1.599 & $2 \rightarrow 1$ & ALMA & 0.77 & 4.36 & $1.1_{-0.2}^{+0.2}$ & $0.7_{-0.3}^{+2.6}$ & $84 \pm 28$ & 12.5 \\
\hline & J0225-324 & 1.600 & $2 \rightarrow 1$ & ALMA & 0.77 & 4.36 & $0.4_{-0.1}^{+0.1}$ & $0.1_{-0.1}^{+0.3}$ & $48 \pm 27$ & 3.1 \\
\hline & J0225-303 & 1.596 & $2 \rightarrow 1$ & ALMA & 0.77 & 4.36 & $2.3_{-0.6}^{+0.6}$ & $4.4_{-0.9}^{+0.8}$ & $3 \pm 3$ & 47.2 \\
\hline \multirow[t]{2}{*}{$\begin{array}{l}\text { XMM-LSS J02182-05102 at } z=1.62 \\
\text { (Rudnick et al. 2017) }\end{array}$} & 30169 & 1.629 & $1 \rightarrow 0$ & ALMA & 1.00 & 4.36 & $0.8_{-0.1}^{+0.1}$ & $16.6_{-4.8}^{+6.8}$ & $12_{-3.5}^{+7.5}$ & 127.1 \\
\hline & 30545 & 1.624 & $1 \rightarrow 0$ & ALMA & 1.00 & 4.36 & $2.6_{-0.1}^{+0.1}$ & $13.8_{-4.0}^{+5.7}$ & $155.6_{-45.4}^{+64.2}$ & 110.7 \\
\hline $\begin{array}{l}\text { SpARCS J033057-284300 at } z=1.63 \\
\text { (Noble et al. 2017) }\end{array}$ & J0330-57 & 1.613 & $2 \rightarrow 1$ & ALMA & 0.77 & 4.36 & $1.4_{-0.6}^{+0.6}$ & $3.3_{-1.5}^{+1.8}$ & $36 \pm 21$ & 38.9 \\
\hline \multirow[t]{4}{*}{$\begin{array}{l}\text { SpARCS J022426-032330 at } z=1.63 \\
\text { (Noble et al. 2017) }\end{array}$} & J0224-3656 & 1.626 & $2 \rightarrow 1$ & ALMA & 0.77 & 4.36 & $1.3_{-0.3}^{+0.3}$ & $10.0_{-4.4}^{+1.2}$ & $43 \pm 20$ & 87.8 \\
\hline & J0224-159 & 1.635 & $2 \rightarrow 1$ & ALMA & 0.77 & 4.36 & $2.0_{-0.5}^{+0.5}$ & $5.9_{-1.1}^{+2.6}$ & $217 \pm 82$ & 60.3 \\
\hline & J0224-3680/3624 & 1.626 & $2 \rightarrow 1$ & ALMA & 0.77 & 4.36 & $4.7_{-0.8}^{+0.8}$ & $9.1_{-1.5}^{+3.5}$ & $68 \pm 24$ & 82.0 \\
\hline & J0224-396/424 & 1.634 & $2 \rightarrow 1$ & ALMA & 0.77 & 4.36 & $5.8_{-0.6}^{+0.6}$ & $16.2_{-2.4}^{+3.7}$ & $166 \pm 60$ & 125.4 \\
\hline \multirow[t]{6}{*}{$\begin{array}{l}\text { Cl J1449+0856 at } z=1.99 \\
\text { (Coogan et al. 2018) }\end{array}$} & 13 & 1.994 & $4 \rightarrow 3$ & ALMA/JVLA & 0.36 & 4.50 & $0.6_{-0.1}^{+0.1}$ & $2.9_{-1.4}^{+2.9}$ & $38 \pm 11$ & 45.7 \\
\hline & 6 & 1.983 & $1 \rightarrow 0$ & ALMA/JVLA & 1.00 & 4.20 & $0.7_{-0.2}^{+0.2}$ & $5.1_{-2.6}^{+5.1}$ & $118 \pm 12$ & 69.7 \\
\hline & N7 & 1.996 & $4 \rightarrow 3$ & ALMA/JVLA & 0.36 & 4.20 & $0.5_{-0.1}^{+0.1}$ & $1.2_{-0.6}^{+1.2}$ & $<26$ & 23.5 \\
\hline & B1 & 1.988 & $1 \rightarrow 0$ & ALMA/JVLA & 1.00 & 3.90 & $0.4_{-0.2}^{+0.2}$ & $6.5_{-3.2}^{+6.4}$ & $57 \pm 12$ & 83.0 \\
\hline & 3 & 1.990 & $4 \rightarrow 3$ & ALMA/JVLA & 0.36 & 4.70 & $0.3_{-0.1}^{+0.1}$ & $2.0_{-1.0}^{+2.0}$ & $<23$ & 35.3 \\
\hline & S7 & 1.982 & $4 \rightarrow 3$ & ALMA/JVLA & 0.36 & - & $0.1_{-0.1}^{+0.1}$ & $3.0_{-1.5}^{+3.0}$ & $<26$ & 47.0 \\
\hline \multirow[t]{4}{*}{$\begin{array}{l}\text { Spider Web proto-cluster at } z=2.16 \\
\text { (Tadaki et al. 2019) }\end{array}$} & 1138.42 & 2.163 & $3 \rightarrow 2$ & ALMA & 0.56 & 10.81 & $1.5_{-0.3}^{+0.3}$ & $0.6_{-0.2}^{+0.2}$ & $41 \pm 12$ & 15.9 \\
\hline & 1138.48 & 2.157 & $3 \rightarrow 2$ & ALMA & 0.56 & 5.55 & $1.8_{-0.1}^{+0.1}$ & $4.5_{-1.2}^{+1.6}$ & $144 \pm 66$ & 69.4 \\
\hline & 1138.54 & 2.148 & $3 \rightarrow 2$ & ALMA & 0.56 & 4.41 & $3.9_{-0.2}^{+0.2}$ & $15.1_{-3.9}^{+5.3}$ & $466 \pm 214$ & 172.1 \\
\hline & 1138.56 & 2.144 & $3 \rightarrow 2$ & ALMA & 0.56 & 9.64 & $1.2_{-0.2}^{+0.2}$ & $0.8_{-0.2}^{+0.3}$ & $36 \pm 11$ & 18.9 \\
\hline \multirow[t]{6}{*}{$\begin{array}{l}\text { USS } 1558003 \text { at } z=2.53 \\
\text { (Tadaki et al. 2019) }\end{array}$} & 1158.43 & 2.528 & $3 \rightarrow 2$ & ALMA & 0.56 & 6.59 & $1.1_{-0.2}^{+0.2}$ & $12.0_{-3.1}^{+4.2}$ & $66 \pm 21$ & 175.0 \\
\hline & 1158.54 & 2.515 & $3 \rightarrow 2$ & ALMA & 0.56 & 12.80 & $0.8_{-0.1}^{+0.1}$ & $2.2_{-0.6}^{+0.1}$ & $67 \pm 20$ & 48.6 \\
\hline & 1158.59 & 2.513 & $3 \rightarrow 2$ & ALMA & 0.56 & 8.69 & $1.7_{-0.1}^{+0.1}$ & $5.2_{-1.4}^{+1.8}$ & $88 \pm 26$ & 92.6 \\
\hline & 1158.64 & 2.529 & $3 \rightarrow 2$ & ALMA & 0.56 & 27.15 & $1.3_{-0.2}^{+0.2}$ & $0.7_{-0.2}^{+0.3}$ & $50 \pm 15$ & 19.7 \\
\hline & 1158.73 & 2.526 & $3 \rightarrow 2$ & ALMA & 0.56 & 13.22 & $0.6_{-0.1}^{+0.1}$ & $2.1_{-0.6}^{+0.8}$ & $106 \pm 31$ & 47.1 \\
\hline & 1158.137 & 2.525 & $3 \rightarrow 2$ & ALMA & 0.56 & 10.70 & $0.5_{-0.1}^{+0.1}$ & $3.2_{-0.8}^{+1.1}$ & $98 \pm 29$ & 64.5 \\
\hline \multirow[t]{2}{*}{$\begin{array}{l}\text { HELAISS02 at } z=2.17 \\
\text { (Gómez-Guijarro et al. 2019) }\end{array}$} & So & 2.173 & $3 \rightarrow 2$ & ALMA & 0.69 & 3.50 & $11.6_{-1.0}^{+1.1}$ & $3.0_{-0.7}^{+1.3}$ & $760 \pm 120$ & 52.0 \\
\hline & S1 & 2.164 & $3 \rightarrow 2$ & ALMA & 0.69 & 9.70 & $0.7_{-0.1}^{+0.2}$ & $11.5_{-2.6}^{+2.6}$ & $269 \pm 37$ & 141.4 \\
\hline
\end{tabular}


Table A.1. continued.

\begin{tabular}{|c|c|c|c|c|c|c|c|c|c|c|}
\hline (Proto-)cluster & Galaxy & $z$ & Transition & Telescope & $r_{\mathrm{J} 1}$ & $\begin{array}{c}\alpha_{\mathrm{CO}} \\
\left(\frac{M_{\odot}}{\mathrm{Kkm} \mathrm{s}^{-1} \mathrm{pc}^{2}}\right)\end{array}$ & $\begin{array}{c}L_{\mathrm{CO}(1 \rightarrow 0)}^{\prime} \\
\left(10^{10} \mathrm{~K} \mathrm{~km} \mathrm{~s}^{-1} \mathrm{pc}^{2}\right)\end{array}$ & $\begin{array}{c}M_{\star} \\
\left(10^{10} M_{\odot}\right)\end{array}$ & $\begin{array}{c}\text { SFR } \\
\left(M_{\odot} / \mathrm{yr}\right)\end{array}$ & $\begin{array}{c}\mathrm{SFR}_{\mathrm{MS}} \\
\left(M_{\odot} / \mathrm{yr}\right)\end{array}$ \\
\hline \multirow[t]{3}{*}{$(1)$} & (2) & (3) & (4) & (5) & $(6)$ & (7) & $(8)$ & $(9)$ & $(10)$ & $(11)$ \\
\hline & S2 & 2.169 & $3 \rightarrow 2$ & ALMA & 0.69 & 5.60 & $2.1_{-0.2}^{+0.2}$ & $14.8_{-3.0}^{+3.0}$ & $302 \pm 49$ & 171.3 \\
\hline & S3 & 2.174 & $3 \rightarrow 2$ & ALMA & 0.69 & 6.70 & $1.0_{-0.2}^{+0.2}$ & $1.3_{-0.4}^{+0.3}$ & $200 \pm 32$ & 27.8 \\
\hline \multirow{5}{*}{$\begin{array}{l}\text { HXMM20 at } z=2.6 \\
\text { (Gómez-Guijarro et al. 2019) }\end{array}$} & & & & & & & & & & \\
\hline & So & 2.602 & $1 \rightarrow 0$ & VLA & 1.00 & 2.90 & $12.0_{-1.8}^{+2.1}$ & $0.8_{-0.1}^{+0.2}$ & $661 \pm 91$ & 22.9 \\
\hline & S1 & 2.598 & $1 \rightarrow 0$ & VLA & 1.00 & 3.70 & $3.5_{-0.9}^{+1.3}$ & $1.3_{-0.1}^{+0.3}$ & $380 \pm 44$ & 33.1 \\
\hline & S2 & 2.602 & $1 \rightarrow 0$ & VLA & 1.00 & 2.40 & $7.2_{-2.0}^{+2.8}$ & $1.1_{-0.2}^{+0.4}$ & $347 \pm 48$ & 29.2 \\
\hline & S4 & 2.597 & $1 \rightarrow 0$ & VLA & 1.00 & 0.80 & $20.2_{-5.6}^{+7.8}$ & $3.2_{-1.7}^{+3.1}$ & $100 \pm 14$ & 65.7 \\
\hline \multirow[t]{4}{*}{$\begin{array}{l}\text { HATLAS J084933+021443 at } z=2.41 \\
\text { (Ivison et al. 2013) }\end{array}$} & HATLAS J084933 W & 2.407 & $1 \rightarrow 0$ & JVLA & 1.00 & 0.80 & $13.8_{-1.7}^{+1.7}$ & $24.0_{-5.8}^{+7.6}$ & 3400 & 279.4 \\
\hline & HATLAS J084933 T & 2.409 & $1 \rightarrow 0$ & JVLA & 1.00 & 0.80 & $15.7_{-2.0}^{+2.0}$ & $10.2_{-2.5}^{+3.3}$ & 1500 & 146.4 \\
\hline & HATLAS J084933 M & 2.418 & $1 \rightarrow 0$ & JVLA & 1.00 & 0.80 & $1.6_{-0.4}^{+0.4}$ & 1.0 & 800 & 25.4 \\
\hline & HATLAS J084933 C & 2.414 & $1 \rightarrow 0$ & JVLA & 1.00 & 0.80 & $2.2_{-0.4}^{+0.4}$ & $2.3_{-1.1}^{+2.3}$ & 640 & 47.5 \\
\hline \multirow[t]{7}{*}{$\begin{array}{l}\text { 4C } 23.56 \text { proto-cluster at } z=2.49 \\
\text { (Lee et al. 2017) }\end{array}$} & HAE3 & 2.486 & $3 \rightarrow 2$ & ALMA & 0.53 & 4.71 & $2.2_{-0.4}^{+0.4}$ & $13.0_{-1.9}^{+1.9}$ & $176 \pm 78$ & 182.3 \\
\hline & HAE4 & 2.478 & $3 \rightarrow 2$ & ALMA & 0.53 & 4.41 & $1.6_{-0.2}^{+0.4}$ & $19.7_{-5.1}^{+5.1}$ & $414 \pm 175$ & 248.9 \\
\hline & HAE5 & 2.487 & $3 \rightarrow 2$ & ALMA & 0.53 & 5.48 & $0.6_{-0.1}^{+0.1}$ & $6.1_{-1.1}^{+1.1}$ & $374 \pm 140$ & 102.7 \\
\hline & HAE8 & 2.486 & $3 \rightarrow 2$ & ALMA & 0.53 & 5.19 & $1.7_{-0.2}^{+0.2}$ & $7.8_{-2.7}^{+2.7}$ & $156 \pm 63$ & 123.7 \\
\hline & HAE9 & 2.486 & $3 \rightarrow 2$ & ALMA & 0.53 & 5.35 & $3.4_{-0.4}^{+0.2}$ & $6.8_{-3.6}^{+3.6}$ & $90 \pm 40$ & 111.5 \\
\hline & HAE10 & 2.486 & $3 \rightarrow 2$ & ALMA & 0.53 & 5.72 & $2.3_{-0.4}^{+0.4}$ & $5.1_{-2.6}^{+2.6}$ & $115 \pm 47$ & 89.6 \\
\hline & HAE16 & 2.483 & $3 \rightarrow 2$ & ALMA & 0.53 & 5.94 & $3.1_{-0.4}^{+0.4}$ & $4.4_{-4.3}^{+4.3}$ & $76 \pm 32$ & 80.0 \\
\hline \multirow[t]{14}{*}{$\begin{array}{l}\text { CLJ001 at } z=2.51 \\
\text { (Wang et al. 2018) }\end{array}$} & 01 & 2.503 & $1 \rightarrow 0$ & JVLA & 1.00 & 4.06 & $2.3_{-0.2}^{+0.2}$ & $22.9_{-6.7}^{+9.4}$ & $610_{-168}^{+232}$ & 282.4 \\
\hline & 02 & 2.507 & $1 \rightarrow 0$ & JVLA & 1.00 & 4.06 & $1.8_{-0.3}^{+0.3}$ & $22.4_{-6.5}^{+9.2}$ & $189_{-72}^{+1117}$ & 278.0 \\
\hline & 03 & 2.514 & $1 \rightarrow 0$ & JVLA & 1.00 & 4.08 & $0.6_{-0.1}^{+0.1}$ & $13.5_{-3.9}^{+5.6}$ & $130_{-48}^{+76}$ & 189.8 \\
\hline & 04 & 2.501 & $1 \rightarrow 0$ & JVLA & 1.00 & 4.08 & $0.6_{-0.1}^{+0.1}$ & $11.5_{-3.4}^{+4.7}$ & $212_{-65}^{+94}$ & 167.0 \\
\hline & 05 & 2.508 & $1 \rightarrow 0$ & JVLA & 1.00 & 4.08 & $2.7_{-0.4}^{+0.1}$ & $10.7_{-3.1}^{+3.4}$ & $474_{-123}^{+655}$ & 159.0 \\
\hline & 06 & 2.494 & $1 \rightarrow 0$ & JVLA & 1.00 & 4.09 & $4.9_{-0.4}^{+0.4}$ & $8.5_{-2.5}^{+3.5}$ & $1275_{-330}^{+445}$ & 132.6 \\
\hline & 07 & 2.505 & $1 \rightarrow 0$ & JVLA & 1.00 & 4.09 & $2.8_{-0.9}^{+0.9}$ & $7.9_{-2.3}^{+3.3}$ & $207_{-70}^{+106}$ & 126.4 \\
\hline & 08 & 2.513 & $1 \rightarrow 0$ & JVLA & 1.00 & 4.10 & $3.2_{-0.3}^{+0.3}$ & $6.8_{-2.0}^{+2.8}$ & $717_{-185}^{+250}$ & 112.3 \\
\hline & 09 & 2.500 & $1 \rightarrow 0$ & JVLA & 1.00 & 4.10 & $0.5_{-0.1}^{+0.1}$ & $6.3_{-1.8}^{+2.6}$ & $161_{-57}^{+88}$ & 106.0 \\
\hline & 10 & 2.506 & $1 \rightarrow 0$ & JVLA & 1.00 & 4.10 & $1.0_{-0.3}^{+0.3}$ & $6.3_{-1.8}^{+2.6}$ & $104_{-45}^{+81}$ & 106.2 \\
\hline & 11 & 2.506 & $1 \rightarrow 0$ & JVLA & 1.00 & 4.10 & $12.7_{-4.2}^{+4.2}$ & $5.4_{-1.6}^{+2.2}$ & $320_{-99}^{+143}$ & 94.0 \\
\hline & 12 & 2.515 & $1 \rightarrow 0$ & JVLA & 1.00 & 4.11 & $0.3_{-0.1}^{+0.1}$ & $4.7_{-1.4}^{+1.9}$ & $22_{-8}^{+13}$ & 85.0 \\
\hline & 13 & 2.505 & $1 \rightarrow 0$ & JVLA & 1.00 & 4.11 & $2.0_{-0.3}^{+0.3}$ & $4.7_{-1.4}^{+1.9}$ & $351_{-130}^{+205}$ & 84.6 \\
\hline & 14 & 2.515 & $1 \rightarrow 0$ & JVLA & 1.00 & 4.12 & $13.3_{-2.7}^{+2.7}$ & $3.5_{-1.0}^{+1.4}$ & $153_{-67}^{+119}$ & 67.7 \\
\hline $\begin{array}{l}\text { SSA22 at } z=3.09 \\
\text { (Bothwell et al. 2013, } \\
\text { Umehata et al. 2015) }\end{array}$ & $\mathrm{J} 221735.15+001537.3$ & 3.096 & $3 \rightarrow 2$ & PdBI & 0.52 & 1.00 & $6.3_{-1.6}^{+2.2}$ & 3.2 & $420_{-80}^{+320}$ & 78.0 \\
\hline \multirow[t]{3}{*}{$\begin{array}{l}\text { GN20 at } z=4.05 \\
\text { (Carilli et al. 2010; Tan et al. 2014) }\end{array}$} & GN20 & 4.055 & $1 \rightarrow 0$ & VLA & 1.00 & 1.30 & $16.2_{-4.6}^{+4.6}$ & 11.0 & $1860 \pm 90$ & 250.8 \\
\hline & GN20.2a & 4.051 & $2 \rightarrow 1$ & EVLA & 0.84 & 2.40 & $9.2_{-5.0}^{+5.0}$ & 3.8 & $800 \pm 70$ & 108.6 \\
\hline & $\mathrm{GN} 20.2 \mathrm{~b}$ & 4.056 & $2 \rightarrow 1$ & EVLA & 0.84 & 2.80 & $2.9_{-1.8}^{+1.8}$ & 11.0 & $690 \pm 100$ & 250.8 \\
\hline $\begin{array}{l}\text { HDF850.1 proto-cluster at } z=5.2 \\
\text { (Walter et al. 2012, } \\
\text { Serjeant \& Marchetti 2014) }\end{array}$ & HDF850.1 & 5.183 & $2 \rightarrow 1$ & JVLA & 0.95 & 0.80 & $4.3_{-1.0}^{+1.0}$ & $13.2_{-5.7}^{+5.7}$ & $850 \pm 255$ & 339.2 \\
\hline $\begin{array}{l}\text { AzTEC-3 proto-cluster at } z=5.3 \\
\text { (Riechers et al. 2010) }\end{array}$ & AzTEC-3 & 5.298 & $2 \rightarrow 1$ & EVLA & 0.88 & 0.80 & $6.6_{-0.9}^{+0.9}$ & $1.0_{-0.2}^{+0.2}$ & 1800 & 43.8 \\
\hline
\end{tabular}


G. Castignani et al.: Molecular gas in distant brightest cluster galaxies

Table A.2. Properties of distant BCG (candidates) observed in CO.

\begin{tabular}{|c|c|c|c|c|c|c|c|c|c|c|}
\hline (Proto-)cluster & Galaxy & $z$ & Transition & Telescope & $r_{\mathrm{J} 1}$ & $\begin{array}{c}\alpha_{\mathrm{CO}} \\
\left(\frac{M_{\odot}}{\mathrm{Kkm} \mathrm{s}^{-1} \mathrm{pc}^{2}}\right)\end{array}$ & $\begin{array}{c}L_{\mathrm{CO}(1 \rightarrow 0)}^{\prime} \\
\left(10^{10} \mathrm{~K} \mathrm{~km} \mathrm{~s}^{-1} \mathrm{pc}^{2}\right)\end{array}$ & $\begin{array}{c}M_{\star} \\
\left(10^{10} M_{\odot}\right)\end{array}$ & $\begin{array}{c}\text { SFR } \\
\left(M_{\odot} / \mathrm{yr}\right)\end{array}$ & $\begin{array}{l}\mathrm{SFR}_{\mathrm{MS}} \\
\left(M_{\odot} / \mathrm{yr}\right)\end{array}$ \\
\hline (1) & $(2)$ & (3) & (4) & $(5)$ & $(6)$ & $(7)$ & $(8)$ & (9) & $(10)$ & $(11)$ \\
\hline $\begin{array}{l}\text { SpARCS } 1049+56 \text { at } z=1.71 \\
\text { (Webb et al. 2015b, 2017) }\end{array}$ & $\mathrm{J} 104922.6+564033$ & 1.709 & $2 \rightarrow 1$ & LMT & 0.85 & 0.80 & $13.8_{-1.2}^{+1.2}$ & 45.0 & $856 \pm 128$ & 281.6 \\
\hline $\begin{array}{l}\text { Cl J1449+0856 at } z=1.99 \\
\text { (Gobat et al. 2011, } \\
\text { Coogan et al. 2018) }\end{array}$ & A1 & 1.990 & $4 \rightarrow 3$ & ALMA & 0.36 & 3.60 & $1.7_{-0.1}^{+0.1}$ & $50.0_{-27.0}^{+27.0}$ & $227 \pm 22$ & 379.2 \\
\hline $\begin{array}{l}\text { Spider Web proto-cluster at } z= \\
\text { (Emonts et al. 2013, 2016) } \\
\text { (Hatch et al. 2008, 2009) }\end{array}$ & MRC 1138-262 & 2.161 & $1 \rightarrow 0$ & ATCA & 1.00 & 4.00 & $5.6_{-1.7}^{+1.7}$ & $110.0_{-20.0}^{+20.0}$ & $142_{-85}^{+1258}$ & 765.2 \\
\hline $\begin{array}{l}\text { Candels-5001 at } z=3.473 \\
\text { (Ginolfi et al. 2017) }\end{array}$ & Candels-5001 & 3.473 & $4 \rightarrow 3$ & ALMA & 0.33 & 10.00 & $1.2_{-0.5}^{+0.6}$ & $1.9_{-0.4}^{+2.6}$ & $214_{-109}^{+223}$ & 55.6 \\
\hline $\begin{array}{l}\text { DES-RG } 399 \text { at } z=0.3 \\
\text { (Castignani et al. 2019) }\end{array}$ & DES-RG 399 & 0.388 & $3 \rightarrow 2$ & IRAM $30 \mathrm{~m}$ & 0.55 & 4.36 & $<0.2$ & 9.1 & $21_{-9}^{+10}$ & 9.8 \\
\hline $\begin{array}{l}\text { DES-RG } 708 \text { at } z=0.6 \\
\text { (Castignani et al. 2019) }\end{array}$ & DES-RG 708 & 0.606 & $3 \rightarrow 2$ & IRAM $30 \mathrm{~m}$ & 0.55 & 4.36 & $<0.6$ & 28.2 & $112_{-58}^{+68}$ & 37.0 \\
\hline $\begin{array}{l}\text { COSMOS-FRI } 16 \text { at } z=0.9 \\
(\text { Castignani et al. 2019) }\end{array}$ & COSMOS-FRI 16 & 0.969 & $4 \rightarrow 3$ & IRAM $30 \mathrm{~m}$ & 0.40 & 4.36 & $<4.3$ & 12.3 & $26_{-7}^{+9}$ & 46.2 \\
\hline $\begin{array}{l}\text { COSMOS-FRI } 31 \text { at } z=0.9 \\
(\text { Castignani et al. 2019) }\end{array}$ & COSMOS-FRI 31 & 0.912 & $4 \rightarrow 3$ & IRAM $30 \mathrm{~m}$ & 0.40 & 4.36 & $<3.6$ & 8.7 & $<42$ & 33.3 \\
\hline $\begin{array}{l}\text { COSMOS-FRI } 70 \text { at } z=2.6 \\
\text { (Castignani et al. 2019) }\end{array}$ & COSMOS-FRI 70 & 2.625 & $7 \rightarrow 6$ & IRAM $30 \mathrm{~m}$ & 0.39 & 4.36 & $<1.5$ & 22.9 & $245_{-112}^{+205}$ & 298.0 \\
\hline $\begin{array}{l}\text { SpARCS J1033+58 at } z=0.43 \\
\text { (This work) }\end{array}$ & $3 \mathrm{C} 244.1$ & 0.430 & $2 \rightarrow 1$ & IRAM $30 \mathrm{~m}$ & 0.80 & 4.36 & $<0.2$ & 10.0 & $281 \pm 218$ & 11.8 \\
\hline $\begin{array}{l}\text { SpARCS J1611+55 at } z=0.91 \\
\text { (This work) }\end{array}$ & SDSS J161112.65+550823.5 & 0.907 & $4 \rightarrow 3$ & IRAM $30 \mathrm{~m}$ & 0.40 & 4.36 & $<0.6$ & 18.0 & $766 \pm 275$ & 53.7 \\
\hline
\end{tabular}

Notes. For the Cl J1449+0856 BCG the reported SFR is derived from the $870 \mu \mathrm{m}$ observer frame flux (Coogan et al. 2018); to estimate $L_{\mathrm{CO}(1 \rightarrow 0)}^{\prime}$, an excitation ratio $r_{41}=0.36$ is assumed, equal to the mean between the ratios of the two Coogan et al. (2018) sources (ID 6, B1) with both $\mathrm{CO}(1 \rightarrow 0)$ and $\mathrm{CO}(4 \rightarrow 3)$ detections. The reported values for $\alpha_{\mathrm{CO}}$ and $L_{\mathrm{CO}(1 \rightarrow 0)}^{\prime}$ imply a molecular gas mass $\sim 10^{10.8} M_{\odot}$, while Coogan et al. (2018) find a dynamical mass equal to $10^{10.3 \pm 0.3} M_{\odot}$. The two estimates are fairly consistent with each other within the uncertainties. For MRC 1138262 we report the $\mathrm{SFR}=142 M_{\odot} \mathrm{yr}^{-1}$ (Emonts et al. 2016), with uncertainties derived assuming maximum and minimum values equal to SFR= $1400 M_{\odot} \mathrm{yr}^{-1}$ (Emonts et al. 2013) and $\mathrm{SFR}=57 M_{\odot} \mathrm{yr}^{-1}$ (Hatch et al. 2008), respectively. 